IASSNS-HEP-99/76

\title{
Quantum Hall Bilayers and the Chiral Sine-Gordon Equation
}

\author{
J. D. Naud ${ }^{1 *}$, Leonid P. Pryadko ${ }^{2}$ and S. L. Sondhi ${ }^{1}$ \\ ${ }^{1}$ Department of Physics, Princeton University, Princeton, NJ 08544, USA \\ ${ }^{2}$ School of Natural Sciences, Institute for Advanced Study, Olden Lane, Princeton, NJ 08540
}

(December 2, 1999)

\begin{abstract}
The edge state theory of a class of symmetric double-layer quantum Hall systems with interlayer electron tunneling reduces to the sum of a free field theory and a field theory of a chiral Bose field with a self-interaction of the sine-Gordon form. We argue that the perturbative renormalization group flow of this chiral sine-Gordon theory is distinct from the standard (non-chiral) sine-Gordon theory, contrary to a previous assertion by Renn, and that the theory is manifestly sensible only at a discrete set of values of the inverse period of the cosine interaction $(\hat{\beta})$. We obtain exact solutions for the spectra and correlation functions of the chiral sine-Gordon theory at the two values of $\hat{\beta}$ at which electron tunneling in bilayers is not irrelevant. Of these, the marginal case $\left(\hat{\beta}^{2}=4\right)$ is of greatest interest: the spectrum of the interacting theory is that of two Majorana fermions with different, dynamically generated, velocities. For the experimentally observed bilayer 331 state at filling factor $1 / 2$, this implies the trifurcation of electrons added to the edge. We also present a method for fermionizing the theory at the discrete points $\left(\hat{\beta}^{2} \in \mathbb{Z}^{+}\right)$ by the introduction of auxiliary degrees of freedom that could prove useful in other problems involving quantum Hall multi-layers.
\end{abstract}

73.40.Hm, 71.10.Pm, 11.25.Hf, 11.10.Hi

Typeset using REVTEX

*Corresponding Author: naud@princeton.edu, 609.258 .5983 (Tel), 609.258.1006 (FAX) 


\section{INTRODUCTION}

This paper is concerned with a chiral version of the celebrated sine-Gordon (SG) field theory in one dimension, in which the standard kinetic term is replaced by the kinetic term for a chiral Bose field. More precisely, we are concerned with the Hamiltonian for a periodic boson of radius $R=1 / \hat{\beta}$,

$$
\mathcal{H}_{\chi S G}[\phi]=\int d x\left[\frac{1}{4 \pi}\left(\partial_{x} \phi\right)^{2}-\kappa \cos (\hat{\beta} \phi)\right],
$$

supplemented by the equal-time commutation relation,

$$
\left[\phi(x), \phi\left(x^{\prime}\right)\right]=i \pi \operatorname{sgn}\left(x-x^{\prime}\right)
$$

appropriate for a chiral Bose field. The Hamiltonian is suggestive of the standard sineGordon theory, but the resemblance is misleading and the physics, as we shall see, is very different on account of the chiral nature of the field. Indeed, a previous analysis of this problem by Renn [1] is in error precisely because of his neglect of the chiral constraint. We will comment further on the difference between the two theories at several points in the main text.

The chief interest of the chiral sine-Gordon $(\chi \mathrm{SG})$ theory is that it arises naturally, as we detail below, in the edge theory of double-layer quantum Hall systems when it is necessary to take account of weak interlayer-tunneling within Wen's chiral bosonic description of the edge dynamics. As such it is the simplest of a family of interacting chiral theories in one dimension that describe the dynamics of multicomponent systems. (Such systems yield non-chiral problems as well, in cases where there are counter-propagating modes, and they have attracted considerable attention 2 14.) These theories are much more constrained than generic non-chiral theories, but they are by no means trivial and they exhibit unusual connections between renormalization group flows and spectral rearrangements. In a sense, they are intermediate in complexity between quantum impurity problems, which can be mapped to chiral problems with interactions localized at a point, and generic one dimensional field theories, and seem worth studying on this account as well. Dijkgraaf has explored some general properties of chiral theories from a conformal field theory viewpoint [5], and several authors have considered particular examples such as the chiral Potts model [6] and the chiral Schwinger model [0]. Finally, the study of chiral field theories is not without interest in another condensed matter context: Ho and Coleman have recently motivated the study of interacting chiral Majorana theories by appealing to the phenomenology of the cuprates and have presented solutions of several models by an interesting "fermionic bootstrap" [8].

We begin with a review of the bosonic formulation of the edge theory of double-layer quantum Hall systems with interlayer tunneling (Section III). For systems with one mode per layer we show that for clean, symmetric realizations, the edge theory separates into the sum of a free boson Hamiltonian and the $\chi \mathrm{SG}$ Hamiltonian with $\hat{\beta}^{2} \in \mathbb{Z}_{\text {even }}^{+}$(the "bosonic" sequence). We argue that the $\chi \mathrm{SG}$ theory is manifestly sensible only for integer values of $\hat{\beta}^{2}$. Layer asymmetry and disorder, which lead to a more complicated dynamics, will be discussed elsewhere [9]. Next we consider the renormalization group flow of the $\chi \mathrm{SG}$ theory, perturbatively in $\lambda$, and argue that it differs from the usual SG theory (Section III). We 
argue that $\hat{\beta}$ is not renormalized and for $\hat{\beta}^{2}=4$ there is a line of fixed points parameterized by $\lambda$. In Section $\mathbb{I D}$ we obtain exact solutions for the spectrum and correlation functions of the $\chi \mathrm{SG}$ theory for $\hat{\beta}^{2}=2$ and $\hat{\beta}^{2}=4$ which are the two members of the bosonic sequence at which the interaction is not irrelevant. At $\hat{\beta}^{2}=2$ we show by "re-bosonization", that the spectrum of the interacting theory is, in the thermodynamic limit, still that of a free chiral boson. Perhaps of greatest interest is our solution by fermionization at $\hat{\beta}^{2}=4$, relevant to the experimentally observed 331 state, where the $\chi \mathrm{SG}$ theory is shown to be equivalent to a theory with two chiral Majorana fermions with dynamically generated distinct velocities. Next we present a method for fermionizing the $\chi \mathrm{SG}$ theory at $\hat{\beta}^{2} \in \mathbb{Z}^{+}$by the introduction of auxiliary bosons (Section $\mathrm{V}$ ). We show that for $\hat{\beta}^{2}=2$ and $\hat{\beta}^{2}=4$ the fermionized theory with auxiliary degrees of freedom can be solved exactly, and after projection onto the $\chi \mathrm{SG}$ Hilbert space we recover the results of Section [V]. In Section V1 we briefly consider the $\chi \mathrm{SG}$ theory at the points $\hat{\beta}^{2} \in \mathbb{Z}_{\text {odd }}^{+}$(the "fermionic" sequence), which do not correspond to any double-layer system. We comment briefly on the difficulties in understanding our results from the kinds of semiclassical considerations that are so useful in the standard sineGordon problem in Section VII. We conclude with a discussion (Section VIII) and several Appendices giving detailed calculations in support of statements made in the text.

\section{EDGE THEORY OF DOUBLE-LAYER SYSTEMS}

\section{A. Double-layer Systems without Tunneling}

We are interested in a system consisting of two parallel layers of 2DEGs (two-dimensional electron gases) in a strong perpendicular magnetic field, with a confining potential that restricts the electrons in each layer to a region with the topology of a disc. The geometry is sketched in Fig. 1. The simplest Abelian quantum Hall states in double-layer systems are well described by the two-component generalization of the Laughlin wavefunction first introduced by Halperin [10]:

$$
\Psi_{m, m^{\prime}, n}\left(\left\{z_{i \alpha}\right\}\right)=\prod_{\alpha<\beta}\left(z_{1 \alpha}-z_{1 \beta}\right)^{m}\left(z_{2 \alpha}-z_{2 \beta}\right)^{m^{\prime}} \prod_{\alpha, \beta}\left(z_{1 \alpha}-z_{2 \beta}\right)^{n} e^{-\sum_{i, \alpha}\left|z_{i \alpha}\right|^{2} / 4},
$$

where $z_{i \alpha}$ is the complex coordinate of electron $\alpha$ in layer $i$, and we work in the symmetric gauge. Here $m$ and $m^{\prime}$ are positive odd integers characterizing the intralayer correlations and $n$ is a non-negative integer specifying interlayer correlations. The filling factor in each layer is 11

$$
\left(\begin{array}{l}
\nu_{1} \\
\nu_{2}
\end{array}\right)=\frac{1}{\left(m m^{\prime}-n^{2}\right)}\left(\begin{array}{c}
m^{\prime}-n \\
m-n
\end{array}\right) .
$$

Following convention we refer to these states by the shorthand $m m^{\prime} n$. 


\section{FIGURES}

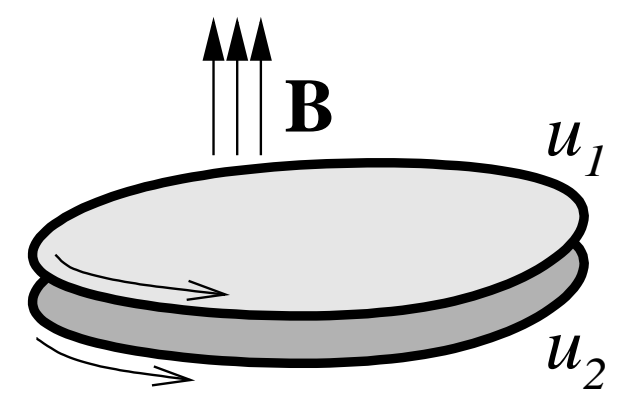

FIG. 1. The overall geometry of a double-layer quantum Hall system, with edge modes in both layers $\left(u_{i}\right)$ propagating in the same direction. The chiral sine-Gordon theory describes the dynamics of the neutral mode in the presence of uniform interedge electron tunneling.

The gapless excitations of the system are confined to the edges of the droplets, and for unreconstructed edges they are described at low-energies by the effective Hamiltonian [12]

$$
\mathcal{H}_{0}=\int_{-L / 2}^{L / 2} d x \frac{1}{4 \pi} V_{i j}: \partial_{x} u_{i} \partial_{x} u_{j}:
$$

where we have assumed the long-range part of the Coulomb interaction is screened. Here $x$ is the coordinate along the edge of length $L$ and normal ordering will be defined below. $V$ is a symmetric matrix whose elements depend on the details of the confining potentials and the interactions at the edge. We require $V$ to be positive definite so that the Hamiltonian is bounded from below. We can parameterize this matrix by

$$
V=\left(\begin{array}{cc}
v & g \\
g & v^{\prime}
\end{array}\right), \quad \text { where } g^{2}<v v^{\prime}
$$

The $u_{i}(t, x)$ are chiral Bose fields with compactification radius $R=1$ (i.e., $u \sim u+2 \pi$ ), which satisfy the equal-time commutation relations:

$$
\left[u_{i}(t, x), u_{j}\left(t, x^{\prime}\right)\right]=i \pi K_{i j} \operatorname{sgn}\left(x-x^{\prime}\right)
$$

where $K$ is a symmetric, integer-valued matrix which characterizes the topological properties of the system and is completely determined by the exponents in the bulk wavefunction 13.

$$
K=\left(\begin{array}{cc}
m & n \\
n & m^{\prime}
\end{array}\right)
$$

From here on, any expression in which the time arguments of the operators are suppressed is to be understood to hold at equal times. Eq. (17) is valid in the limit of large $L$; if we retained the full $L$ dependence the r.h.s. would be periodic $\left(\bmod 2 \pi i K_{i j}\right)$ in $\left(x-x^{\prime}\right)$ with period $L$.

Since the chiral Bose fields are compact, we can introduce the integer-valued Hermitian topological charge operators

$$
\mathcal{N}_{i}=\frac{1}{2 \pi} \int_{-L / 2}^{L / 2} d x \partial_{x} u_{i}(x)
$$


which by Eq. (đ) obey:

$$
\left[\mathcal{N}_{i}, u_{j}(x)\right]=i K_{i j}, \quad\left[\mathcal{N}_{i}, \mathcal{N}_{j}\right]=0
$$

For a chiral boson with a radius $R \neq 1$, the coefficient of the integral in Eq. (9) would be $1 /(2 \pi R)$. The electric charge density on the edge of layer $i, \rho_{i}(x)$, and the total charge, $Q_{i}$, are related to the corresponding topological quantities by:

$$
\rho_{i}(x)=\frac{1}{2 \pi} K_{i j}^{-1} \partial_{x} u_{j}(x), \quad Q_{i}=K_{i j}^{-1} \mathcal{N}_{j}
$$

The vertex operators which create an edge quasiparticle or an edge electron in layer $i$ are, respectively,

$$
\Psi_{q p, i}^{\dagger}(x) \propto e^{-i K_{i j}^{-1} u_{j}(x)}, \quad \Psi_{i}^{\dagger}(x) \propto e^{-i u_{i}(x)} .
$$

These operators change the topological and electrical charges according to

$$
\begin{aligned}
{\left[\mathcal{N}_{i}, \Psi_{q p, j}^{\dagger}\right] } & =\delta_{i j} \Psi_{q p, j}^{\dagger}, \quad\left[Q_{i}, \Psi_{q p, j}^{\dagger}\right]=K_{i j}^{-1} \Psi_{q p, j}^{\dagger}, \\
{\left[\mathcal{N}_{i}, \Psi_{j}^{\dagger}\right] } & =K_{i j} \Psi_{j}^{\dagger}, \quad\left[Q_{i}, \Psi_{j}^{\dagger}\right]=\delta_{i j} \Psi_{j}^{\dagger} .
\end{aligned}
$$

The physical meaning of the edge quasiparticle creation operator is as follows. When a quasihole is created in the bulk fluid (e.g., by the insertion of a flux quantum), the charge depleted from the vicinity of the quasihole accumulates at the boundary of the droplet. In the somewhat artificial theoretical construction in which the "bulk" and "edge" of the droplet are considered distinct, this accumulation appears as the addition of a fractional amount of charge to the edge theory, which is accomplished by the edge quasiparticle creation operator.

If we consider the action of the vertex operators (12) in a two-dimensional integer lattice in the $\mathcal{N}_{1} \mathcal{N}_{2}$-plane, the quasiparticle creation operators can be identified with the basis vectors of this lattice: $\Psi_{q p, 1}^{\dagger} \rightarrow(1,0), \Psi_{q p, 2}^{\dagger} \rightarrow(0,1)$. The vectors corresponding to the electron creation operators, $\Psi_{1}^{\dagger} \rightarrow(m, n), \Psi_{2}^{\dagger} \rightarrow\left(n, m^{\prime}\right)$, are the basis vectors of an "electron sublattice" consisting of all states connected to one another by adding or removing only electrons from the edges [14]. The number of distinct electron sublattices is equal to the volume of the unit cell spanned by the electron vectors: $m m^{\prime}-n^{2}$. We shall refer to these as different sectors of the edge theory. Note that the discrete spectrum of the topological charges also places restrictions on the allowed vertex operators, as we describe below. An example of the topological charge lattice is shown in Fig. 2 for the 331 state (i.e., $m=m^{\prime}=3, n=1$ ). 


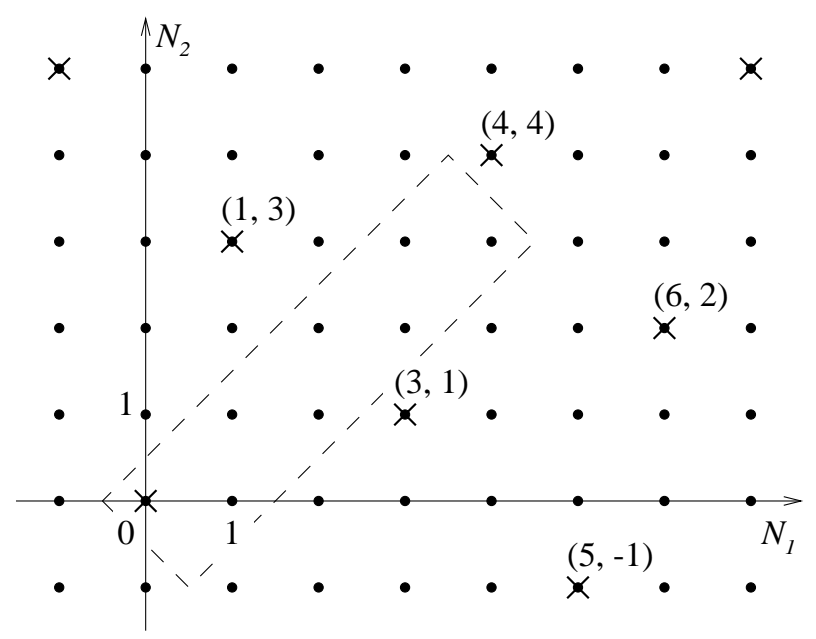

FIG. 2. The topological charge lattice in the $\mathcal{N}_{1} \mathcal{N}_{2}$-plane for the double-layer 331 state. The crosses denote points in the electron sublattice (i.e., sector) containing the origin. The unit cell of this sublattice is enclosed by the dashed line.

From Eqns. (10) and (12) we find

$$
\begin{aligned}
\Psi_{i}^{\dagger}(x+L) & =\Psi_{i}^{\dagger}(x) e^{-2 \pi i \mathcal{N}_{i}} e^{-i \pi K_{i i}} \\
\Psi_{q p, i}^{\dagger}(x+L) & =\Psi_{q p, i}^{\dagger}(x) e^{-2 \pi i K_{i j}^{-1} \mathcal{N}_{j}} e^{-i \pi K_{i i}^{-1}} .
\end{aligned}
$$

Since $\mathcal{N}_{i} \in \mathbb{Z}$ the boundary conditions on the electron operators are independent of the values of the topological charges. Within the sector containing the origin, $\mathcal{N}_{i}=0$, the same statement holds for the quasiparticle operators. However, in other sectors (e.g., the state with a single quasiparticle in layer $1, \mathcal{N}_{1}=1, \mathcal{N}_{2}=0$ ) the quasiparticle operator acquires an additional phase as it is taken around the edge. Since creating a quasiparticle at the edge requires the creation of a quasihole in the bulk as noted above, this additional phase can be interpreted as the Aharonov-Bohm phase due to the flux creating the bulk quasihole [15].

We are interested in the case where the two edge modes have the same chirality. Since $V$ is positive definite, the chirality of the edge bosons is determined by the signs of the eigenvalues of $K$. Therefore we shall restrict our discussion to the "maximally chiral" case in which both eigenvalues of $K$ are positive, which requires $n^{2}<m m^{\prime}$. (The requirement that the filling factor in each layer be positive then gives $n<m, m^{\prime}$.) In this case, the scaling dimensions of vertex operators are independent of $V$ [16]. Furthermore we shall consider only the symmetric case: $m=m^{\prime}$ and $v=v^{\prime}$. Our result in Section III for the lowest-order perturbative RG flow of the tunneling amplitude applies for the case $v \neq v^{\prime}$ and can easily be extended to states with $m \neq m^{\prime}$, but the mapping to the chiral sine-Gordon theory exists only in the symmetric case, as we demonstrate below.

The Hamiltonian, Eq. (5), can be simplified by performing an orthogonal transformation on the chiral Bose fields to diagonalize $K$ and $V$, followed by a rescaling to bring $K$ to the identity. The combined transformation to the new fields $\phi_{\mathrm{c}}, \phi_{\mathrm{n}}$ is:

$$
\left(\begin{array}{l}
u_{1} \\
u_{2}
\end{array}\right)=\frac{1}{\sqrt{2}}\left(\begin{array}{cc}
\sqrt{m+n} & -\sqrt{m-n} \\
\sqrt{m+n} & \sqrt{m-n}
\end{array}\right)\left(\begin{array}{c}
\phi_{\mathrm{c}} \\
\phi_{\mathrm{n}}
\end{array}\right)
$$


We will refer to $\phi_{\mathrm{c}}, \phi_{\mathrm{n}}$ as the charged and neutral edge modes, respectively, since the topological charge of $\phi_{\mathrm{c}}$ is proportional to the total electric charge on the two edges while the topological charge of $\phi_{\mathrm{n}}$ is proportional to the electric charge difference between the edges. When we write $\phi_{i}$, the index i runs over the two values $\mathrm{i}=\mathrm{c}, \mathrm{n}$. In terms of the new fields, the commutators are now independent and conventionally normalized:

$$
\left[\phi_{\mathrm{i}}(x), \phi_{\mathrm{j}}\left(x^{\prime}\right)\right]=i \pi \delta_{\mathrm{ij}} \operatorname{sgn}\left(x-x^{\prime}\right) .
$$

The mode expansions of the rotated chiral bosons are

$$
\phi_{\mathrm{i}}(x)=\frac{2 \pi}{L} N_{\mathrm{i}} R_{\mathrm{i}} x-\frac{1}{R_{\mathrm{i}}} \chi_{\mathrm{i}}+\sum_{r=1}^{\infty} \frac{1}{\sqrt{r}} e^{-k_{r} a / 2}\left(e^{-i k_{r} x} b_{\mathrm{i} r}^{\dagger}+e^{i k_{r} x} b_{\mathrm{i} r}\right),
$$

where $k_{r} \equiv 2 \pi r / L, R_{\mathrm{i}}$ are the compactification radii of the rotated fields, $N_{\mathrm{i}}$ are the new topological charges, $\chi_{\mathrm{i}}$ are the conjugate Hermitian phase operators, $b_{\mathrm{i} r}$ are bosonic annihilation operators, and $a$ is a short-distance cutoff. The only nonvanishing commutators are

$$
\left[b_{\mathrm{i} r}, b_{\mathrm{j} s}^{\dagger}\right]=\delta_{\mathrm{ij}} \delta_{r s}, \quad\left[\chi_{\mathrm{i}}, N_{\mathrm{j}}\right]=i \delta_{\mathrm{ij}}
$$

The normal ordering introduced in Eq. (5) is defined with respect to the bosonic oscillators $b_{\mathrm{i} r}, b_{\mathrm{i} r}^{\dagger}$. Up to this point we have neglected normal ordering when writing vertex operators. Using Eq. (17) one can show that normal ordering produces a multiplicative renormalization of vertex operators which to lowest order in $a / L$ gives

$$
: e^{i \alpha \phi_{\mathrm{i}}(x)}:=\left(\frac{L}{2 \pi a}\right)^{\Delta(\alpha)} e^{i \alpha \phi_{\mathrm{i}}(x)}
$$

where $\Delta(\alpha) \equiv \alpha^{2} / 2$ is the scaling dimension of the operator, and also its conformal spin since $\phi_{\mathrm{i}}$ is a chiral field.

Using Eqns. (15) and (17) we can express the Hamiltonian (5) in terms of the modes of the uncoupled fields:

$$
\mathcal{H}_{0}=\int_{-L / 2}^{L / 2} d x\left[\frac{1}{4 \pi} v_{\mathrm{i}}:\left(\partial_{x} \phi_{\mathrm{i}}\right)^{2}:\right]=\frac{\pi v_{\mathrm{i}}}{L}\left(R_{\mathrm{i}} N_{\mathrm{i}}\right)^{2}+\frac{2 \pi}{L} \sum_{r=1}^{\infty} r v_{\mathrm{i}} b_{\mathrm{i} r}^{\dagger} b_{\mathrm{i} r},
$$

where the velocities of the eigenmodes are $v_{\mathrm{c}, \mathrm{n}}=(m \pm n)(v \pm g)$. The structure of the Hilbert space is now clear. There are an infinite number of states $\left|N_{\mathrm{c}}, N_{\mathrm{n}}\right\rangle$ which are eigenstates of the topological charge operators and which are annihilated by $b_{\mathrm{i} r}$ for $\mathrm{i}=\mathrm{c}, \mathrm{n}$, and $r>0$. This infinite set of oscillator vacua is divided into a finite number of sectors as defined above. The (zero-energy) ground state is $|0,0\rangle$. Note that because Eq. (15) defines a homogeneous linear transformation, the states $\left|N_{\mathrm{c}}, N_{\mathrm{n}}\right\rangle$ are also vacua for the oscillator modes of the original bosons $\left(u_{i}\right)$.

The values of the radii $R_{\mathrm{i}}$ are determined by the requirement that the new winding number operators $N_{\mathrm{i}}$ have integer eigenvalues. From the zero-mode piece of the inverse of the transformation (15) we find 


$$
R_{\mathrm{c}} N_{\mathrm{c}}=\frac{1}{\sqrt{2(m+n)}}\left(\mathcal{N}_{1}+\mathcal{N}_{2}\right), \quad R_{\mathrm{n}} N_{\mathrm{n}}=\frac{1}{\sqrt{2(m-n)}}\left(\mathcal{N}_{2}-\mathcal{N}_{1}\right)
$$

Since $\mathcal{N}_{i} \in \mathbb{Z}$ we see that the choice $R_{\mathrm{c}}=1 / \sqrt{2(m+n)}, R_{\mathrm{n}}=1 / \sqrt{2(m-n)}$ restricts $N_{\mathrm{c}}=\mathcal{N}_{1}+\mathcal{N}_{2}, N_{\mathrm{n}}=\mathcal{N}_{2}-\mathcal{N}_{1}$ to be integers. From this relation we also see that $N_{\mathrm{c}}$ and $N_{\mathrm{n}}$ are not independent since they must satisfy the "gluing condition" $N_{\mathrm{c}}+N_{\mathrm{n}} \in \mathbb{Z}_{\text {even }}$ in order to describe the original $\mathcal{N}_{1} \mathcal{N}_{2}$ integer lattice. If one considers the electron sublattice containing the ground state (i.e., the origin $\mathcal{N}_{i}=N_{\mathrm{i}}=0$ ), then one may choose a unit cell such that the $m^{2}-n^{2}$ points in distinct electron sublattices are labeled by

$$
\left\{\left(N_{\mathrm{c}}, N_{\mathrm{n}}\right) \mid N_{\mathrm{n}}=0,-1, \ldots-(m-n-1) ; N_{\mathrm{c}}-F_{N_{\mathrm{n}}}=0,2, \ldots 2(m+n-1)\right\},
$$

where the offset $F_{N_{\mathrm{n}}} \equiv\left[1-(-1)^{N_{\mathrm{n}}}\right] / 2$ is zero if $N_{\mathrm{n}}$ is even and one if $N_{\mathrm{n}}$ is odd. From this it is clear that the $m^{2}-n^{2}$ distinct sectors of the full edge theory correspond to $f_{\mathrm{n}} \equiv m-n$ sectors for the neutral boson and $f_{\mathrm{c}} \equiv m+n$ sectors for the charged boson. The requirement that the vertex operator $e^{-i \alpha \phi_{i}}$ changes the topological charge $N_{\mathrm{i}}$ by an integer restricts the allowed values of $\alpha$ to the set $R_{\mathrm{i}} \mathbb{Z}$. In terms of their action in the topological charge lattice, the vertex operators with $\alpha=j R_{\mathrm{i}}$ for $j=1,2, \ldots\left(f_{\mathrm{i}}-1\right)$ take us between the different sectors of the $\phi_{\mathrm{i}}$ field.

The partition function and correlation functions can readily be obtained since $\mathcal{H}_{0}$ is quadratic. If we imagine that our system is connected to a reservoir (e.g., a normal metal) such that the edge can gain or lose electrons without disturbing the bulk state, then the physically meaningful partition function is obtained by summing only over states in a single sector. Going between sectors requires the addition of an edge quasiparticle, which must be accompanied by the creation of a bulk quasihole, for which there is a finite energy gap. At finite length $L$ and inverse temperature $\beta$ the partition function in the ground state sector (i.e., the sector containing $|0,0\rangle$ ) can be written

$$
\begin{aligned}
Z_{0}(\beta)=\operatorname{Tr}\left(e^{-\beta \mathcal{H}_{0}}\right)= & \chi_{R_{\mathrm{c}}}^{(2(m+n), 0)}\left(q_{\mathrm{c}}\right) \chi_{R_{\mathrm{n}}}^{(2(m-n), 0)}\left(q_{\mathrm{n}}\right) \\
& +\chi_{R_{\mathrm{c}}}^{(2(m+n), m+n)}\left(q_{\mathrm{c}}\right) \chi_{R_{\mathrm{n}}}^{(2(m-n), m-n)}\left(q_{\mathrm{n}}\right),
\end{aligned}
$$

where $q_{\mathrm{i}} \equiv e^{-2 \pi \beta v_{\mathrm{i}} / L}$, and

$$
\chi_{R}^{(r, s)}(q) \equiv \frac{1}{\varphi(q)} \sum_{p \in \mathbb{Z}} q^{\frac{1}{2}[R(r p+s)]^{2}}, \quad \text { with } \quad \varphi(q) \equiv \prod_{k=1}^{\infty}\left(1-q^{k}\right),
$$

The function $\chi_{R}^{(r, s)}(q)$ is the character of a radius $R$ compact chiral boson whose topological charge is restricted to the set $r \mathbb{Z}+s$. We note in passing the well known result that $\chi_{1}^{(1,0)}(q)$ is equivalent to the character of a chiral Dirac fermion on a circle with antiperiodic boundary conditions [i.e., $\psi(x+L)=-\psi(x)]$ [17, 18].

When writing correlation functions we work at zero temperature, in imaginary time $\tau=i t$, and in the limit $L \rightarrow \infty, a \rightarrow 0$. Since this involves evaluating ground state expectation values of operators that preserve the parity of $N_{\mathrm{c}}+N_{\mathrm{n}}$, the gluing condition does not complicate the calculation. We choose a non-standard engineering dimension for the electron operators so that their correlation functions are well behaved in the limit $L \rightarrow \infty$, 


$$
\Psi_{i}(x)=\frac{1}{L^{m / 2}} e^{i u_{i}(x)} .
$$

The electron propagator is

$$
\begin{aligned}
-\mathcal{G}_{i j}(\tau, x) & \equiv\left\langle T_{\tau}: \Psi_{i}(\tau, x):: \Psi_{j}^{\dagger}(0,0):\right\rangle \\
& =\frac{\delta_{i j}}{\left[2 \pi\left(v_{\mathrm{c}} \tau-i x\right)\right]^{\frac{(m+n)}{2}}\left[2 \pi\left(v_{n} \tau-i x\right)\right]^{\frac{(m-n)}{2}}} .
\end{aligned}
$$

The equal-time Green's function has a spatial dependence $\mathcal{G}_{i i}(0, x) \sim x^{-m}$ which clearly signals Luttinger liquid behavior for $m \neq 1$. The two-point function of the density operator (11) is:

$$
\begin{aligned}
\mathcal{D}_{i j}(\tau, x) & \equiv\left\langle T_{\tau} \rho_{i}(\tau, x) \rho_{j}(0)\right\rangle \\
& =\frac{1}{2(m+n)} \frac{1}{\left[2 \pi\left(v_{\mathrm{c}} \tau-i x\right)\right]^{2}}+\frac{(-1)^{i+j}}{2(m-n)} \frac{1}{\left[2 \pi\left(v_{\mathrm{n}} \tau-i x\right)\right]^{2}} .
\end{aligned}
$$

Note that the off-diagonal term, $\mathcal{D}_{12}=\mathcal{D}_{21}$, is non-zero even in the absence of tunneling; it vanishes only when there are no interlayer correlations in the bulk $(n=0)$ and no interlayer interactions at the edge $(g=0)$.

\section{B. Interlayer Tunneling and the Quantum Chiral sine-Gordon Equation}

We now consider adding interlayer electron tunneling to the edge theory. Interlayer quasiparticle tunneling at the edge is not considered since the addition of a quasiparticle to the edge requires the creation of a bulk quasihole, and hence it is not a low-energy excitation. The electron operators defined above have the property that

$$
e^{i u_{i}(x)} e^{i u_{j}\left(x^{\prime}\right)}=e^{i u_{j}\left(x^{\prime}\right)} e^{i u_{i}(x)} e^{i \pi \operatorname{sgn}\left(x^{\prime}-x\right) K_{i j}}, \quad \text { for } \quad x \neq x^{\prime} .
$$

If $K_{12}=n$ is even, this implies that the electron operators in different layers commute rather than anticommute. This can be remedied by the introduction of Klein factors constructed from the topological charges in such a way that the tunneling Hamiltonian defined below is not modified. The details of this procedure are given in Appendix A.

The Hamiltonian describing interlayer single-electron tunneling is:

$$
\mathcal{H}_{1}=\lambda_{0} \int_{-L / 2}^{L / 2} d x\left[: \Psi_{1}(x) \Psi_{2}^{\dagger}(x):+ \text { h.c. }\right]
$$

where $\lambda_{0}$ is the tunneling amplitude, which is assumed to be uniform along the edge. Using the transformation (15) and removing the normal ordering with Eq. (19), the full Hamilto-

nian $\mathcal{H}=\mathcal{H}_{0}+\mathcal{H}_{1}$ can be expressed in terms of the rotated Bose fields as

$$
\begin{aligned}
\mathcal{H} & =\int_{-L / 2}^{L / 2} d x\left[\frac{1}{4 \pi} v_{\mathrm{c}}:\left(\partial_{x} \phi_{\mathrm{c}}\right)^{2}:+\frac{1}{4 \pi} v_{\mathrm{n}}:\left(\partial_{x} \phi_{\mathrm{n}}\right)^{2}:+\frac{2 \lambda}{(2 \pi a)^{\hat{\beta}^{2} / 2}} \cos \left(\hat{\beta} \phi_{\mathrm{n}}\right)\right] \\
& \equiv \mathcal{H}_{F}\left[\phi_{\mathrm{c}}\right]+\mathcal{H}_{\chi S G}^{\hat{\beta}^{2}}\left[\phi_{\mathrm{n}}\right]
\end{aligned}
$$


where $\hat{\beta} \equiv \sqrt{2(m-n)}$, and $\lambda \equiv \lambda_{0} L^{-n}$ has length dimension $\left(\hat{\beta}^{2} / 2-2\right)$. Note that because of our normalization of the bosonic fields, $\hat{\beta}$ differs from the inverse period conventionally used in the SG theory by a factor of $\sqrt{2 \pi}$.

The Hamiltonian separates into two commuting pieces, a free chiral boson Hamiltonian $\left(\mathcal{H}_{F}\right)$ for the field $\phi_{\mathrm{c}}$, and a chiral sine-Gordon Hamiltonian $\left(\mathcal{H}_{\chi S G}^{\hat{\beta}^{2}}\right)$ for the field $\phi_{\mathrm{n}}$. If we had taken $v \neq v^{\prime}$ there would be a cross term in the Hamiltonian of the form $\partial_{x} \phi_{\mathrm{c}} \partial_{x} \phi_{\mathrm{n}}$, while if we had taken $m \neq m^{\prime}$ the two pieces of the Hamiltonian would not commute. For the case of $v \neq v^{\prime}$ we could perform a further orthogonal transformation on the Bose fields which would preserve the commutation relations (16) and eliminate the cross term in the Hamiltonian, but then the tunneling term would involve both transformed fields [9].

An important property of the tunneling Hamiltonian, $\mathcal{H}_{1}$, is that it commutes with $\mathcal{H}_{0}$. This follows from Eq. (C11) in Appendix C. Physically, it is a consequence of the fact that the translationally invariant perturbation conserves momentum, which in a chiral system with a single velocity is proportional to the energy. It follows that first-order degenerate perturbation theory for this system is exact, and therefore the exact eigenvalues of $\mathcal{H}_{\chi S G}^{\hat{\beta}^{2}}$ depend linearly on $\lambda$.

Since the zero-energy ground state of $\mathcal{H}_{0},|0,0\rangle$, is non-degenerate, another consequence of the vanishing commutator, $\left[\mathcal{H}_{0}, \mathcal{H}_{1}\right]=0$, is that $|0,0\rangle$ is also an exact eigenstate of the tunneling Hamiltonian. In fact, $\mathcal{H}_{1}$ annihilates the ground state of $\mathcal{H}_{0}$, as we now show. Using the mode expansion (17) we can write

$$
\begin{aligned}
\frac{1}{a^{\hat{\beta}^{2} / 2}} \cos \left(\hat{\beta} \phi_{\mathrm{n}}(x)\right)= & \frac{1}{2}\left(\frac{2 \pi}{L}\right)^{\hat{\beta}^{2} / 2} e^{-i \pi \hat{\beta}^{2} x / L} \\
& \times\left[e^{-i \hat{\beta} \chi_{\mathrm{n}} / R_{\mathrm{n}}} e^{i \hat{\beta} \phi_{\mathrm{n}}^{(+)}(x)} e^{i \hat{\beta} \phi_{\mathrm{n}}^{(-)}(x)} e^{2 \pi i \hat{\beta} R_{\mathrm{n}} N_{\mathrm{n}} x / L}+(\hat{\beta} \rightarrow-\hat{\beta})\right]
\end{aligned}
$$

where we have introduced

$$
\phi_{\mathrm{n}}^{(-)}(x) \equiv \sum_{r=1}^{\infty} \frac{1}{\sqrt{r}} e^{-k_{r} a / 2} e^{i k_{r} x} b_{\mathrm{n} r}, \quad \phi_{\mathrm{n}}^{(+)} \equiv\left(\phi_{\mathrm{n}}^{(-)}\right)^{\dagger} .
$$

If we act on the state $|0,0\rangle$ with Eq. (31) and imagine expanding the exponentials involving $N_{\mathrm{n}}$ and $\phi_{\mathrm{n}}^{(-)}$we see that only the zeroth-order term survives. We then have

$$
\begin{aligned}
& \int_{-L / 2}^{L / 2} d x \frac{1}{a^{\hat{\beta}^{2} / 2}} \cos \left(\hat{\beta} \phi_{\mathrm{n}}(x)\right)|0,0\rangle=\left\{\frac{1}{2}\left(\frac{2 \pi}{L}\right)^{\hat{\beta}^{2} / 2} e^{-i \hat{\beta} \chi_{\mathrm{n}} / R_{\mathrm{n}}} \int_{-L / 2}^{L / 2} d x e^{-i \pi \hat{\beta}^{2} x / L}\right. \\
& \left.\times\left[1+\sum_{r=1}^{\infty} \sum_{s_{1}=1}^{\infty} \cdots \sum_{s_{r}=1}^{\infty} e^{(-2 \pi i x / L) \sum_{j=1}^{r} s_{j}} \frac{(i \hat{\beta})^{r} e^{(-\pi a / L) \sum_{j=1}^{r} s_{j}}}{r ! \sqrt{\prod_{j=1}^{r} s_{j}}} \prod_{j=1}^{r} b_{\mathrm{n} s_{j}}^{\dagger}\right]+(\hat{\beta} \rightarrow-\hat{\beta})\right\}|0,0\rangle,
\end{aligned}
$$

where we have expanded the exponential of $\phi_{\mathrm{n}}^{(+)}$. The $x$ integral vanishes in every term ( since $\hat{\beta}^{2} \in \mathbb{Z}_{\text {even }}^{+}$), establishing that $|0,0\rangle$ is an exact zero-energy eigenstate of $\mathcal{H}_{0}+\mathcal{H}_{1}$. Of course this does not imply that this state is the ground state of the full Hamiltonian.

The chiral sine-Gordon Hamiltonian we have arrived at from the bilayer quantum Hall system has the properties: $\hat{\beta}^{2} \in \mathbb{Z}_{\text {even }}^{+}$, and $R_{\mathrm{n}}=1 / \hat{\beta}$. It is natural to ask if the $\chi \mathrm{SG}$ theory can be sensibly defined for more general choices of these parameters. As discussed above, the 
requirement that $\cos \left(\hat{\beta} \phi_{\mathrm{n}}\right)$ changes the winding number by an integer gives the restriction $\hat{\beta} / R_{\mathrm{n}}=p$ for some $p \in \mathbb{Z}$. Next consider the behavior of the perturbation under $x \rightarrow x+L$,

$$
\cos \hat{\beta} \phi_{\mathrm{n}}(x+L)=\frac{1}{2} e^{-i \pi \hat{\beta}^{2}}\left(e^{i \hat{\beta} \phi_{\mathrm{n}}(x)} e^{2 \pi i \hat{\beta} R_{\mathrm{n}} N_{\mathrm{n}}}+e^{-i \hat{\beta} \phi_{\mathrm{n}}(x)} e^{-2 \pi i \hat{\beta} R_{\mathrm{n}} N_{\mathrm{n}}}\right) .
$$

Since this operator appears in the Hamiltonian we demand that the periodicity be independent of the state on which it acts, which implies $\hat{\beta} R_{\mathrm{n}}=p^{\prime}$ for some integer $p^{\prime}$. Combining these two conditions yields $\hat{\beta}^{2}=p p^{\prime}$, and $R_{\mathrm{n}}^{2}=p^{\prime} / p$. Without loss of generality we can take $p$ and $p^{\prime}$ to be positive. We find that in addition to the case $\hat{\beta}^{2} \in \mathbb{Z}_{\text {even }}^{+}$previously considered, there is the case $\hat{\beta}^{2} \in \mathbb{Z}_{\text {odd }}^{+}$. We shall refer to these as the "bosonic" and "fermionic" sequences, respectively, since from Eq. (34) the perturbation is periodic for even $\hat{\beta}^{2}$ and antiperiodic for odd $\hat{\beta}^{2}$.

To reiterate, for non-integer values of $\hat{\beta}^{2}$ there does not exist a choice of the radius $R_{\mathrm{n}}$ that ensures that the Hamiltonian density changes the topological charge by an integer and has a periodicity under $x \rightarrow x+L$ that is independent of the state on which it acts. For a given value of $\hat{\beta}^{2}$, different choices of the radius do not correspond to distinct theories. If for a fixed value of $\hat{\beta}^{2}$ we take the minimum value of $R_{\mathrm{n}}$, we recover the condition $R_{\mathrm{n}}=1 / \hat{\beta}$. The other allowed radii at this value of $\hat{\beta}^{2}$ are integer multiples of this minimum radius and by Eq. (24), they correspond to restricting the allowed values of the topological charge in the theory with the minimum $R_{\mathrm{n}}$.

\section{RG FLOW OF THE CHIRAL SINE-GORDON THEORY}

At the end of the previous section we found that the $\chi \mathrm{SG}$ theory appears to be sensibly defined only at discrete values of $\hat{\beta}^{2}$. This suggests that $\hat{\beta}$ does not flow under a renormalization group transformation. In this section we examine this question directly. We work in the limit $L \rightarrow \infty$ and consider the Euclidean action of the $\chi$ SG theory [19],

$$
S_{\chi S G}[\phi]=\int d x d \tau\left(\frac{1}{4 \pi}\left[i \partial_{\tau} \phi \partial_{x} \phi+\left(\partial_{x} \phi\right)^{2}\right]+\frac{\hat{\lambda}}{(2 \pi a)^{2}} \cos (\hat{\beta} \phi)\right),
$$

where $\hat{\lambda} \equiv 2 \lambda(2 \pi a)^{2-\hat{\beta}^{2} / 2}$ is the dimensionless coupling and in this section we set $v_{\mathrm{n}}=1$ for simplicity. Here and in the remainder of the paper we will suppress the subscript on the neutral boson, i.e., $\phi \equiv \phi_{\mathrm{n}}$. Recall that the action of the standard (i.e., non-chiral) sine-Gordon theory is [20]:

$$
S_{S G}[\phi]=\int d x d \tau\left(\frac{1}{4 \pi}\left[\left(\partial_{\tau} \phi\right)^{2}+\left(\partial_{x} \phi\right)^{2}\right]+\frac{\hat{\lambda}}{a^{2}} \cos (\hat{\beta} \phi)\right) .
$$

The RG behavior of $S_{S G}$ is the well known Kosterlitz-Thouless flow 21 24]:

$$
\frac{d \hat{\lambda}}{d \ell}=\left(2-\frac{\hat{\beta}^{2}}{2}\right) \hat{\lambda}+\mathcal{O}\left(\hat{\lambda}^{2}\right), \quad \frac{d \hat{\beta}^{2}}{d \ell}=-c_{0} \hat{\lambda}^{2}+\mathcal{O}\left(\hat{\lambda}^{3}\right),
$$

where the UV momentum cutoff decreases as $\ell$ increases, and the value of the constant $c_{0}>0$ is non-universal. In Ref. [1], Renn argues that $S_{\chi S G}$ can be mapped into $S_{S G}$ by a 
coordinate transformation, which would imply that the $\mathrm{RG}$ flow of the $\chi \mathrm{SG}$ theory is also given by Eq. (37). This argument is flawed since at the level of the free action, $\hat{\lambda}=0$, the two theories clearly have different numbers of degrees of freedom (the non-chiral boson can be written as the sum of two chiral bosons) and hence cannot be related by a change of coordinates. The classical (real-time) equation of motion for a free chiral boson which follows from the Minkowski version of the quadratic part of Eq. (35) is $\left(\partial_{x} \partial_{t}+\partial_{x}^{2}\right) \phi=0$, which has a general solution $\phi(t, x)=\phi_{R}(x-t)+\vartheta(t)$ where $\vartheta(t)$ is a gauge degree of freedom. The coordinate transformation Renn proposed amounts to promoting this unphysical degree of freedom into a left-moving boson, which when combined with the physical right-moving degree of freedom, $\phi_{R}$, gives a non-chiral boson.

A standard perturbative, momentum-shell RG analysis for $S_{\chi S G}$ gives to first order in $\hat{\lambda}$

$$
\frac{d \hat{\lambda}}{d \ell}=\left(2-\frac{\hat{\beta}^{2}}{2}\right) \hat{\lambda}, \quad \frac{d \hat{\beta}^{2}}{d \ell}=0 .
$$

We see that tunneling is irrelevant for $\hat{\beta}^{2}>4$ and relevant for $\hat{\beta}^{2}<4$. The irrelevant region includes all fractional quantum Hall states without interlayer correlations, i.e., $n=0$ and $m=3,5, \ldots$ Whether an $\mathcal{O}\left(\hat{\lambda}^{2}\right)$ term in the flow of $\hat{\beta}^{2}$ is present (as it is for the non-chiral theory) is a difficult question to answer unambiguously using a Wilsonian RG. Indeed, to rigorously obtain the $-c_{0} \hat{\lambda}^{2}$ term in Eq. (37) from a momentum-shell RG one must use a smooth cutoff [21]. Only because of the Euclidean (rotational) symmetry of the free part of $S_{S G}$ can this term be found simply by using an ad-hoc regularization of the divergent integral that would be rendered finite if one employed smooth momentum-space slicing [21]24]. The free chiral boson action does not possess this symmetry, and even continuous regularizations lead to different results depending on the details of the particular cutoff procedure.

Nevertheless, we now argue that there is no flow in $\hat{\beta}$ for the $\chi \mathrm{SG}$ theory, i.e., $d \hat{\beta}^{2} / d \ell=0$ to all orders in $\hat{\lambda}$. Consider calculating the correlation functions of some vertex operator perturbatively in $\hat{\lambda}$, for example the two-point function:

$$
\begin{aligned}
\mu^{\alpha^{2}}\left\langle: e^{i \alpha \phi\left(z_{1}\right)}:: e^{-i \alpha \phi\left(z_{2}\right)}:\right\rangle= & \frac{1}{\left(z_{1}-z_{2}\right)^{\alpha^{2}}}\left\{1+\frac{\hat{\lambda}^{2}}{8 a^{4-\hat{\beta}^{2}}} \int d^{2} w_{1} d^{2} w_{2} \frac{1}{\left(w_{1}-w_{2}\right)^{\hat{\beta}^{2}}}\right. \\
& \left.\times\left[\left(\frac{\left(z_{1}-w_{1}\right)\left(z_{2}-w_{2}\right)}{\left(z_{1}-w_{2}\right)\left(z_{2}-w_{1}\right)}\right)^{\alpha \hat{\beta}}+\left(w_{1} \leftrightarrow w_{2}\right)-2\right]+\mathcal{O}\left(\hat{\lambda}^{4}\right)\right\}
\end{aligned}
$$

where $z \equiv \tau-i x$, and $\mu$ is an infrared momentum cutoff needed since we are working at $L \rightarrow \infty$. We see that for general $\alpha$ the multi-valuedness of the correlation function is altered by the perturbative corrections. This should be contrasted with the standard SG theory, where $(w-z)$ in Eq. (39) is replaced by $|w-z|$ and correlation functions of all vertex operators are single-valued at every order in $\hat{\lambda}$ for arbitrary $\hat{\beta}$. Note however that for the set of allowed vertex operators defined in Section $\mathbb{I}$, $\alpha=p / \hat{\beta}$ where $p \in \mathbb{Z}$, we see from Eq. (39) that the $\mathcal{O}\left(\hat{\lambda}^{2}\right)$ term has the same multi-valuedness as a function of $\left(z-z^{\prime}\right)$ as the leading-order term. This property holds for $N$-point correlation functions $(N>2)$ and for higher-order corrections in $\hat{\lambda}$, since the factor of $\hat{\beta}$ cancels whenever we contract an external vertex operator $\left[e^{i(p / \hat{\beta}) \phi\left(z_{i}\right)}\right]$ with an internal one $\left[e^{i \hat{\beta} \phi\left(w_{i}\right)}\right]$. It is conceivable that the theory 
could be defined at non-integer values of $\hat{\beta}^{2}$ for this subset of operators, but we still contend that there is no flow in $\hat{\beta}$.

Suppose we begin with some bare values of the parameters, $\hat{\lambda}_{0}$ and $\hat{\beta}_{0}$, defined for a theory with a UV momentum cutoff $\Lambda \sim 1 / a$ and imagine running the $\mathrm{RG}$ so that the cutoff is reduced to $\Lambda / s$ where $s>1$, and $\hat{\lambda}_{0} \rightarrow \hat{\lambda}(s), \hat{\beta}_{0} \rightarrow \hat{\beta}(s)$. We allow for a field renormalization by defining $\phi^{\prime}\left(z^{\prime}\right) \equiv Z_{\phi}^{-1}(s) \phi(z)$, where $z^{\prime}=z / s$. The covariance of correlation functions under the RG flow implies that for $\left|z_{1}^{\prime}-z_{2}^{\prime}\right| \gg(\Lambda / s)^{-1}$,

$$
\left\langle: e^{i \alpha \phi\left(z_{1}\right)}:: e^{-i \alpha \phi\left(z_{2}\right)}:\right\rangle_{\hat{\beta}_{0}}=\left\langle: e^{i \alpha Z_{\phi}(s) \phi^{\prime}\left(z_{1}^{\prime}\right)}:: e^{-i \alpha Z_{\phi}(s) \phi^{\prime}\left(z_{2}^{\prime}\right)}:\right\rangle_{\hat{\beta}(s)},
$$

where the 1.h.s. is evaluated in the bare theory and the r.h.s. in the renormalized theory. A key point is that any flow in $\hat{\beta}$ is governed by the field renormalization: $\hat{\beta}(s)=Z_{\phi}(s) \hat{\beta}_{0}$. If we choose $\alpha=p / \hat{\beta}_{0}$ for some integer $p$, so that the perturbative corrections on the l.h.s. of Eq. (40) do not change the multi-valuedness of the leading-order term, then the requirement that the same thing be true on the r.h.s., along with the relations $Z_{\phi}(1)=1, \hat{\beta}(1)=\hat{\beta}_{0}$, implies $\alpha Z_{\phi}(s)=p / \hat{\beta}(s)$. Eliminating $Z_{\phi}(s)$ and $\alpha$ from this equation yields $\hat{\beta}(s)=\hat{\beta}_{0}$, i.e., $\hat{\beta}$ does not flow. This argument for the non-renormalization of $\hat{\beta}$ can at best be viewed as heuristic; its validity is intricately connected to the way the RG transformation is defined. We believe that at least the weaker statement that there exists an $R G$ procedure for which there is no flow in $\hat{\beta}$, is true. The exact solutions of the theory at $\hat{\beta}^{2}=2$ and $\hat{\beta}^{2}=4$ presented in the next section support this claim.

In light of the argument that $d \hat{\beta} / d \ell=0$, the flow in $\hat{\lambda}$ at the lowest-order marginal point, $\hat{\beta}^{2}=4$, will be governed by the higher-order terms in the first expression in Eq. (38). Because of the complexity of the higher-order bosonic perturbation theory, we shall postpone this calculation until we find a fermionic representation of the theory in the next section. We will find that at this value of $\hat{\beta}$ the tunneling perturbation is exactly marginal, i.e., there is a line of fixed points parameterized by $\hat{\lambda}$. The RG flows of the standard SG and $\chi \mathrm{SG}$ theories are shown in Fig. 3 .
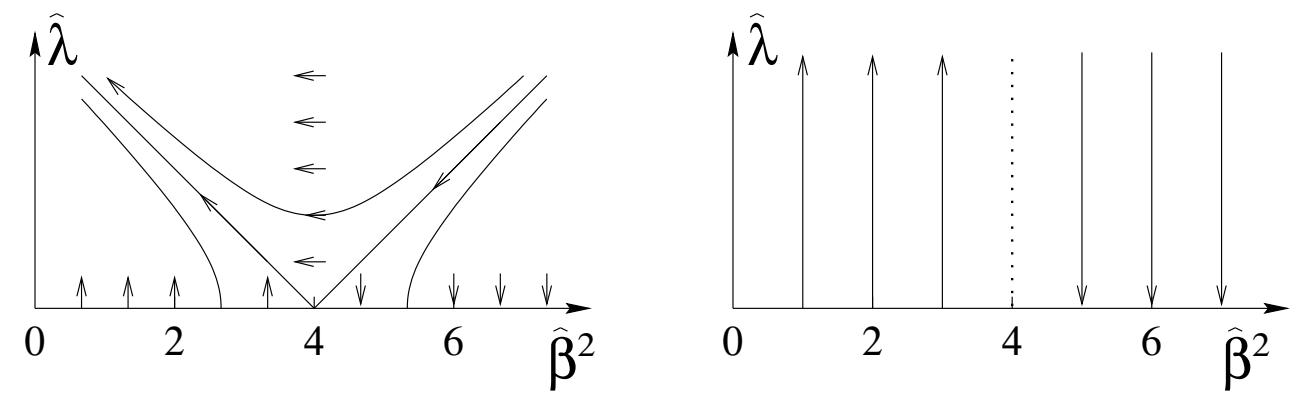

FIG. 3. Left: the perturbative RG flow for the standard SG theory. Both $\hat{\lambda}$ and $\hat{\beta}^{2}$ are renormalized. Right: the perturbative RG flow for the $\chi \mathrm{SG}$ theory. $\hat{\beta}$ does not flow and there is a line of fixed points at $\hat{\beta}^{2}=4$ (dots).

\section{EXACT SOLUTIONS OF THE CHIRAL SINE-GORDON THEORY}

In this section we present exact solutions of the $\chi \mathrm{SG}$ theory at the points $\hat{\beta}^{2}=2$ and $\hat{\beta}^{2}=4$. 


\section{A. Relevant Tunneling, $\hat{\beta}^{2}=2$}

At the point $\hat{\beta}^{2}=2$, which corresponds to $m-n=1$, the cosine term is relevant. There is an infinite sequence of $K$ matrices describing maximally chiral edges which satisfy this condition: $\left(m, m^{\prime}, n\right)=(1,1,0)+r(2,2,2), r=0,1, \ldots$ The discussion here also applies to the bosonic states $\left(m, m^{\prime}, n\right)=(2,2,1)+r(2,2,2)$.

At this value of $\hat{\beta}$ the $\chi \mathrm{SG}$ Hamiltonian is

$$
\mathcal{H}_{\chi S G}^{\hat{\beta}^{2}=2}=\int_{-L / 2}^{L / 2} d x\left[\frac{1}{4 \pi} v_{\mathrm{n}}:\left(\partial_{x} \phi\right)^{2}:+\frac{\lambda}{2 \pi a}\left(e^{i \sqrt{2} \phi}+\text { h.c. }\right)\right]
$$

where $\phi(x)$ is a radius $R_{\mathrm{n}}=1 / \sqrt{2}$ chiral boson. A similar problem, with $\lambda$ replaced by a Gaussian random variable, appears in Kane, Fisher, and Polchinski's work on the disordered, single-layer, $\nu=2 / 3$ edge [2]. A special property of a radius $1 / \sqrt{2}$ free chiral boson is that its character (24) can be expressed as:

$$
\chi_{1 / \sqrt{2}}^{(1,0)}(q)=\chi_{s u(2)_{1}}^{(0)}(q)+\chi_{s u(2)_{1}}^{(1 / 2)}(q)
$$

where $\chi_{s u(2)_{1}}^{(j)}(q)$ is the character of the spin- $j$ irreducible representation of the $\widehat{s u}(2)_{1}$ KacMoody (KM) algebra 25]. Indeed, the trio of dimension one operators $\partial_{x} \phi, e^{i \sqrt{2} \phi}, e^{-i \sqrt{2} \phi}$ are related to the $\widehat{s u}(2)_{1}$ generators, $J^{a}(x), a=x, y, z$, by 25

$$
J^{z}(x)=\frac{1}{2 \pi \sqrt{2}} \partial_{x} \phi(x), \quad J^{ \pm}(x)=J^{x} \pm i J^{y}=\frac{1}{2 \pi a} e^{ \pm i \sqrt{2} \phi(x)} .
$$

In terms of the Fourier components

$$
J_{n}^{a} \equiv \int_{-L / 2}^{L / 2} d x J^{a}(x) e^{-2 \pi i n x / L}, \quad n \in \mathbb{Z}
$$

the algebra is

$$
\left[J_{n}^{a}, J_{m}^{b}\right]=\frac{n}{2} \delta^{a b} \delta_{n+m, 0}+i \epsilon^{a b c} J_{n+m}^{c} .
$$

With the help of Eq. (43) the Hamiltonian (41) can be expressed in terms of currents

$$
\mathcal{H}_{\chi S G}^{\hat{\beta}^{2}=2}=\int_{-L / 2}^{L / 2} d x\left\{2 \pi v_{\mathrm{n}}:\left[J^{z}(x)\right]^{2}:+2 \lambda J^{x}(x)\right\}
$$

We now use an identity valid for any state $|\gamma\rangle$ in the Hilbert space

$$
\int_{-L / 2}^{L / 2} d x:\left[J^{z}(x)\right]^{2}:|\gamma\rangle=\int_{-L / 2}^{L / 2} d x \frac{1}{3}:[\mathbf{J}(x)]^{2}:|\gamma\rangle
$$

to write Eq. (46) as:

$$
\mathcal{H}_{\chi S G}^{\hat{\beta}^{2}=2}=\int_{-L / 2}^{L / 2} d x\left\{\frac{2 \pi v_{\mathrm{n}}}{3}:[\mathbf{J}(x)]^{2}:+2 \lambda J^{x}(x)\right\} .
$$


One way to prove Eq. (47) is to note that the operators $J_{n}^{z}$ obey a $\hat{u}(1)$ subalgebra of the $\widehat{s u}(2)_{1}$ algebra generated by the $J_{n}^{a}$ operators. We can construct two families of operators

$$
L_{n} \equiv \frac{1}{3}: J_{m}^{a} J_{n-m}^{a}:, \quad L_{n}^{(z)} \equiv: J_{m}^{z} J_{n-m}^{z}:
$$

each of which obey $c=1$ Virasoro algebras. Since the $J_{n}^{z}$ generate a subalgebra we have $\left[L_{n}, L_{m}^{(z)}\right]=\left[L_{n}^{(z)}, L_{m}^{(z)}\right]$, and therefore by the standard Goddard-Kent-Olive (GKO) coset construction [26] the operators $L_{n}^{(0)} \equiv L_{n}-L_{n}^{(z)}$ obey a $c=0$ Virasoro algebra. It is well known that the only unitary representation of the $c=0$ Virasoro algebra is trivial, i.e., $L_{n}^{(0)}|\gamma\rangle=0$ for all $n$ [27. We thus conclude that $L_{n}|\gamma\rangle=L_{n}^{(z)}|\gamma\rangle$ for any state in the Hilbert space, and for $n=0$ we obtain Eq. (47).

Given any matrix $R \in S O(3)$, we see from Eq. (45) that the currents $\tilde{J}^{a}(x) \equiv R^{a b} J^{b}(x)$ also obey an $\widehat{s u}(2)_{1} \mathrm{KM}$ algebra since $\delta^{a b}$ and $\epsilon^{a b c}$ are $S O(3)$-invariant tensors. If we make the particular choice $R^{a b}=\delta^{a y} \delta^{b y}+\delta^{a z} \delta^{b x}-\delta^{a x} \delta^{b z}$, which corresponds to a rotation by $\pi / 2$ about the $y$-axis, and express the rotated $\widehat{s u}(2)_{1}$ currents in terms of a new radius $R=1 / \sqrt{2}$ chiral boson, $\theta(x)$ (i.e., $\tilde{J}^{z}(x)=\left[\partial_{x} \theta(x)\right] /(2 \pi \sqrt{2})$, etc.) then from Eqns. (47) and (48) we have

$$
\begin{aligned}
\mathcal{H}_{\chi S G}^{\hat{\beta}^{2}=2} & =\int_{-L / 2}^{L / 2} d x\left\{2 \pi v_{\mathrm{n}}:\left[\tilde{J}^{z}(x)\right]^{2}:+2 \lambda \tilde{J}^{z}(x)\right\} \\
& =\int_{-L / 2}^{L / 2} d x\left[\frac{1}{4 \pi} v_{\mathrm{n}}:\left(\partial_{x} \theta\right)^{2}:+\frac{\sqrt{2} \lambda}{2 \pi} \partial_{x} \theta\right] .
\end{aligned}
$$

The mapping between Eqns. (41) and (50), when expressed in terms of the two Bose fields corresponds to the non-linear transformation

$$
\partial_{x} \theta(x)=\frac{\sqrt{2}}{a} \cos (\sqrt{2} \phi(x)), \quad \partial_{x} \phi(x)=-\frac{\sqrt{2}}{a} \cos (\sqrt{2} \theta(x)) .
$$

While we could have taken the first expression in this equation as our definition of $\theta(x)$ and proceeded by showing this implies $:\left(\partial_{x} \phi\right)^{2}:=:\left(\partial_{x} \theta\right)^{2}:$, the detour through the KM algebras is more deductive.

We have succeeded in writing the Hamiltonian as a quadratic form in terms of a single chiral boson. The tunneling term is linear in the topological charge of the field $\theta(x)$, and thus it is clear that the $\lambda=0$ ground state, which is annihilated by the perturbation, is not the ground state for $\lambda \neq 0$. However, since the $\lambda=0$ ground state remains an exact eigenstate of Eq. (50) for all $\lambda$ [see Eq. (33)] we conclude that zero-temperature perturbation theory in $\lambda$ gives incorrect results. In terms of first-order degenerate perturbation theory for the low-lying eigenvalues of $\mathcal{H}_{\chi S G}^{\hat{\beta}^{2}=2}$, this is a consequence of a level crossing for $\lambda=\mathcal{O}(1 / L)$.

Several authors have claimed that a gap is generated when the perturbation is relevant [1.28. It is clear from Eq. (50) that this is not the case. We still have a (gapless) chiral boson, whose topological charge sectors are reorganized in energy. The energy spectrum of the winding number mode for two different values of $\lambda$ is shown in Fig. $⿴$. For the points $\lambda=\left(\pi v_{\mathrm{n}} / L\right) M$, where $M \in \mathbb{Z}$, this reorganization can be reduced to an overall shift in the energy spectrum by defining $\tilde{N}_{\theta}=N_{\theta}+M$. The subtlety is that while we have a gapless mode for $\lambda=0(\phi(x))$ and a gapless mode for $\lambda \neq 0(\theta(x))$, the modes are non-trivially 
connected even for arbitrarily small $\lambda$. We see from the relation, Eq. (51), that exciting modes of the original boson involves the operator $\partial_{x} \phi \sim \cos (\sqrt{2} \theta)$, which changes $N_{\theta}$ and hence involves an energy of order $\lambda$ even for small momentum excitations. Therefore the original field appears massive, whereas the number of gapless modes is unchanged.

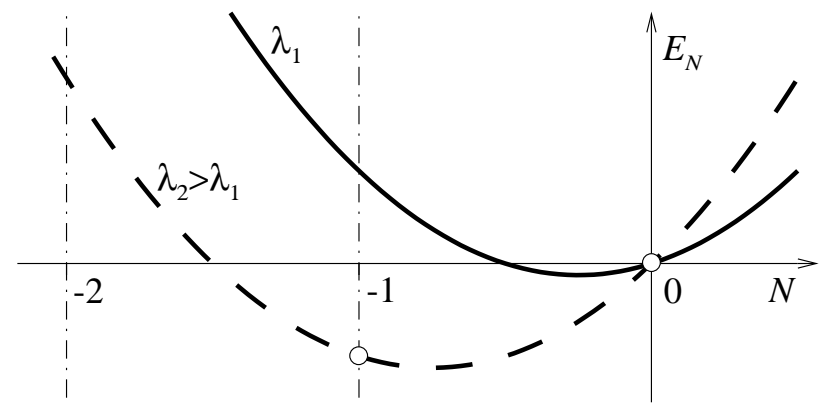

FIG. 4. Energy spectrum for the topological charge $(N)$ of the field $\theta(x)$ at two different values of $\lambda$. The open circles indicate the ground state. For small $\lambda$ (solid line) the ground state is unchanged, while at a larger value of $\lambda$ (dashed line) the ground state has a non-zero winding number.

From Eq. (50) we can immediately write down the partition function for the $\chi \mathrm{SG}$ theory in isolation

$$
Z_{\chi S G}^{\hat{\beta}^{2}=2}(\beta)=\frac{1}{\varphi\left(q_{\mathrm{n}}\right)} \sum_{N \in \mathbb{Z}} e^{-\beta \lambda N} q_{\mathrm{n}}^{N^{2} / 4} .
$$

To construct the partition function of the full edge theory we need to consider gluing conditions. Recall from Section \ that we need to separate states according to their $N_{\mathrm{n}}$-parity, as in the partition function at $\lambda=0$ (23). For $\lambda \neq 0$, eigenstates of the Hamiltonian are no longer eigenstates of $N_{\mathrm{n}}$. However, we see from Eqns. (43) and (44), that $N_{\mathrm{n}}=2 J_{0}^{z}$ and similarly $N_{\theta}=2 \tilde{J}_{0}^{z}$, where $N_{\theta}$ is the winding number operator of the field $\theta(x)$. Therefore, states in the spin-0 (1/2) representation of the $J_{n}^{a}$ algebra have $N_{\mathrm{n}}$ even (odd). Under the rotation from $J^{a}$ to $\widetilde{J}^{a}$ the two representations do not mix, and hence eigenstates of Eq. (50) which have $N_{\theta}$ even (odd) are linear combinations of $N_{\mathrm{n}}$ eigenstates, all of which have even (odd) $N_{\mathrm{n}}$ eigenvalues. Thus we can write the partition function including the charged mode as:

$$
Z^{\hat{\beta}^{2}=2}(\beta)=\chi_{R_{\mathrm{c}}}^{(2(m+n), 0)}\left(q_{\mathrm{c}}\right) Z_{\chi S G}^{\hat{\beta}^{2}=2, \text { even }}(\beta)+\chi_{R_{\mathrm{c}}}^{(2(m+n), m+n)}\left(q_{\mathrm{c}}\right) Z_{\chi S G}^{\hat{\beta}^{2}=2, \text { odd }}(\beta),
$$

where $Z_{\chi S G}^{\hat{\beta}^{2}=2 \text {, even }}$ is given by Eq. (52) with the restriction $N \in \mathbb{Z}_{\text {even }}$, and similarly for $Z_{\chi S G}^{\hat{\beta}^{2}=2, \text { odd }}$.

We now turn to the computation of correlation functions. We define the tunneling operator as the Hamiltonian density of the tunneling perturbation

$$
\Theta(x) \equiv \frac{2}{(2 \pi a)^{\hat{\beta}^{2} / 2}} \cos [\hat{\beta} \phi(x)],
$$


and denote its two-point correlation function by $\mathcal{T}(\tau, x) \equiv\left\langle T_{\tau} \Theta(\tau, x) \Theta(0)\right\rangle$. Using transformations (15) and (51) we can express the charge density (11), and tunneling operator (54), in terms of the fields $\phi_{\mathrm{c}}$ and $\theta$,

$$
\begin{aligned}
\rho_{1,2}(x) & =\frac{1}{\sqrt{8 \pi^{2}(2 m-1)}} \partial_{x} \phi_{c}(x) \pm \frac{1}{2 \pi a} \cos \sqrt{2} \theta(x), \\
\Theta(x) & =\frac{1}{\pi \sqrt{2}} \partial_{x} \theta(x) .
\end{aligned}
$$

Since the Hamiltonian $\mathcal{H}_{F}\left[\phi_{\mathrm{c}}\right]+\mathcal{H}_{\chi S G}^{\hat{\beta}^{2}=2}[\theta]$ is quadratic in these fields we find

$$
\begin{aligned}
\mathcal{D}_{i j}(\tau, x) & =\frac{1}{2(2 m-1)} \frac{1}{\left[2 \pi\left(v_{\mathrm{c}} \tau-i x\right)\right]^{2}}+\frac{(-1)^{i+j}}{2} \frac{\cos \left(2 \lambda x / v_{\mathrm{n}}\right)}{\left[2 \pi\left(v_{\mathrm{n}} \tau-i x\right)\right]^{2}}, \\
\mathcal{T}(\tau, x) & =\frac{2}{\left[2 \pi\left(v_{\mathrm{n}} \tau-i x\right)\right]^{2}}+\left(\frac{\lambda}{\pi v_{\mathrm{n}}}\right)^{2} .
\end{aligned}
$$

The part of the density-density correlation function arising from the neutral mode is spatially modulated when tunneling is present, while the two-point correlation function of the tunneling operator acquires a constant piece. Both of these are a result of the non-zero topological charge of the $\theta$ field in the ground state.

The calculation of the electron propagator is slightly more involved. From Eqns. (15) and (25) we find that for $m-n=1$ the electron operators can be written

$$
\Psi_{1,2}=\frac{1}{L^{m / 2}} e^{i\left(\sqrt{m-\frac{1}{2}} \phi_{\mathrm{c}} \mp \frac{1}{\sqrt{2}} \phi\right)}
$$

and therefore they cannot be readily expressed in terms of $\phi_{\mathrm{c}}$ and $\theta$. However, from this expression it is clear that the single-electron Green's function factorizes:

$$
\begin{aligned}
-\mathcal{G}_{i j}(\tau, x)= & \frac{1}{L^{m / 2}}\left\langle e^{i \sqrt{m-\frac{1}{2}} \phi_{\mathrm{c}}(\tau, x)} e^{-i \sqrt{m-\frac{1}{2}} \phi_{\mathrm{c}}(0)}\right\rangle_{\mathcal{H}_{F}\left[\phi_{\mathrm{c}}\right]} \\
& \times\left\langle e^{\frac{i}{\sqrt{2}}(-1)^{i} \phi(\tau, x)} e^{-\frac{i}{\sqrt{2}}(-1)^{j} \phi(0)}\right\rangle_{\mathcal{H}_{\chi S G}^{\hat{\beta}^{2}=2}} .
\end{aligned}
$$

The correlation function involving the $\phi$ field is the same for all values of $m$ and $n$ that satisfy $m-n=1$. The correlation function involving $\phi_{\mathrm{c}}$ can be computed for all $m$ and $n$ since $\mathcal{H}_{F}\left[\phi_{c}\right]$ is quadratic.

For the special case $m=1, n=0$ the l.h.s. of Eq. (60) can be calculated exactly as we now demonstrate [29]. For the uncorrelated integer 110 state there exists a chiral fermion description of the edge theory including tunneling, $\mathcal{H}_{0}+\mathcal{H}_{1}$ :

$$
\mathcal{H}^{(110)}=\int_{-L / 2}^{L / 2} d x:\left[-i v \psi_{i}^{\dagger} \partial_{x} \psi_{i}+2 \pi g \psi_{1}^{\dagger} \psi_{1} \psi_{2}^{\dagger} \psi_{2}-\lambda\left(\psi_{2}^{\dagger} \psi_{1}+\text { h.c. }\right)\right]: \text {. }
$$

The details in going from the bosonic form of the Hamiltonian (30) to the above fermionic form are discussed in Section $\nabla$. If we define $\psi_{ \pm}(x)=\left(\psi_{1}(x) \pm \psi_{2}(x)\right) / \sqrt{2}$, Eq. (61) becomes 


$$
\mathcal{H}^{(110)}=\int_{-L / 2}^{L / 2} d x:\left[-i v\left(\psi_{+}^{\dagger} \partial_{x} \psi_{+}+\psi_{-}^{\dagger} \partial_{x} \psi_{-}\right)+2 \pi g \psi_{+}^{\dagger} \psi_{+} \psi_{-}^{\dagger} \psi_{-}-\lambda\left(\psi_{+}^{\dagger} \psi_{+}-\psi_{-}^{\dagger} \psi_{-}\right)\right]: .
$$

This Hamiltonian can be brought to a quadratic form by bosonizing according to $\psi_{ \pm}(x)=$ $e^{i \phi_{ \pm}(x)} / \sqrt{2 \pi a}$. The resulting quadratic form is diagonalized by defining $\theta_{+} \equiv\left(\phi_{+}+\phi_{-}\right) / \sqrt{2}$, and $\theta_{-} \equiv\left(\phi_{+}-\phi_{-}\right) / \sqrt{2}+\sqrt{2} \lambda x / v_{\mathrm{n}}$. The exact single-electron Green's function is then readily found:

$$
-\mathcal{G}_{i j}^{(110)}(\tau, x)=\frac{\delta_{i j} \cos \left(\lambda x / v_{\mathrm{n}}\right)-\left(1-\delta_{i j}\right) i \sin \left(\lambda x / v_{\mathrm{n}}\right)}{\sqrt{2 \pi\left(v_{\mathrm{c}} \tau-i x\right) 2 \pi\left(v_{\mathrm{n}} \tau-i x\right)}} .
$$

We can thus use Eqns. (60) and (63) to find the single-electron Green's function for all states in the $m-n=1$ sequence:

$$
-\mathcal{G}_{i j}(\tau, x)=\frac{\delta_{i j} \cos \left(\lambda x / v_{\mathrm{n}}\right)-\left(1-\delta_{i j}\right) i \sin \left(\lambda x / v_{\mathrm{n}}\right)}{\left[2 \pi\left(v_{\mathrm{c}} \tau-i x\right)\right]^{m-\frac{1}{2}} \sqrt{2 \pi\left(v_{\mathrm{n}} \tau-i x\right)}}
$$

the only modification being the exponent of the piece corresponding to the charged boson.

\section{B. Marginal Tunneling, $\hat{\beta}^{2}=4$}

The point $\hat{\beta}^{2}=4$, at which the cosine term is marginal, corresponds to the case $m-n=2$. There is again an infinite sequence of $K$ matrices describing maximally chiral edges which satisfy this condition: $\left(m, m^{\prime}, n\right)=(3,3,1)+r(2,2,2), r=0,1, \ldots$ The results here also apply to the bosonic states $\left(m, m^{\prime}, n\right)=(2,2,0)+r(2,2,2)$.

At this value of $\hat{\beta}$ the $\chi \mathrm{SG}$ Hamiltonian is

$$
\mathcal{H}_{\chi S G}^{\hat{\beta}^{2}=4}=\int_{-L / 2}^{L / 2} d x\left[\frac{1}{4 \pi} v_{\mathrm{n}}:\left(\partial_{x} \phi\right)^{2}:+\frac{\lambda}{(2 \pi a)^{2}}\left(e^{2 i \phi}+\text { h.c. }\right)\right],
$$

where $\phi(x)$ is a radius $R_{\mathrm{n}}=1 / 2$ chiral boson. If we restrict ourselves to the sector of the edge theory that contains the ground state, then we find that $N_{\mathrm{n}} \in \mathbb{Z}_{\text {even }}$, since adding an electron to layer 1(2) changes $N_{\mathrm{n}}$ by $\mp 2$. From the expression for the compact chiral boson character (24), it is clear that a radius $1 / 2$ boson restricted to even topological charges is equivalent to a radius 1 boson with no restriction $\left[i . e ., \chi_{1 / 2}^{(2,0)}(q)=\chi_{1}^{(1,0)}(q)\right]$. Hence, for this sector of the edge theory we can take $\phi(x)$ to have radius 1 and thus define a chiral Dirac fermion $\psi(x) \equiv e^{i \phi(x)} / \sqrt{2 \pi a}$. This result, that the edge theory of double-layer states with $m-n=2$ contains a chiral Dirac fermion was previously established in the wavefunction approach by Milovanović and Read [14].

To find a fermionic representation of the tunneling term, we first consider bringing together two chiral Dirac annihilation operators

$$
\psi(x) \psi(x+\epsilon)=\epsilon \psi(x) \partial_{x} \psi(x)+\mathcal{O}\left(\epsilon^{2}\right),
$$


where the zeroth-order term vanishes by Fermi statistics. We next consider bringing together the bosonized representation of the Fermi fields

$$
\begin{aligned}
\frac{1}{L}: e^{i \phi(x)}:: e^{i \phi(x+\epsilon)}:= & \frac{2 \pi a}{L^{2}}: e^{2 i \phi(x)}: \\
& +\frac{2 \pi i \epsilon}{L^{2}}\left\{: e^{2 i \phi(x)}:-a:\left[\partial_{x} \phi(x)+\frac{3 \pi}{L}\right] e^{2 i \phi(x)}:\right\}+\mathcal{O}\left(\epsilon^{2}, a^{2}\right) .
\end{aligned}
$$

Since the terms on the r.h.s. are normal ordered, we can safely take the limit $a \rightarrow 0$, and compare the order $\epsilon$ terms in Eqns. (66) and (67) to obtain the identity

$$
\psi(x) \partial_{x} \psi(x)=\frac{i}{2 \pi a^{2}} e^{2 i \phi(x)} .
$$

We verified the validity of this formula by checking that the $N$-point correlation functions of the operator on the l.h.s. (and its Hermitian conjugate) calculated in the free Fermi theory are identical to the $N$-point correlation functions of the operator on the r.h.s. (and its Hermitian conjugate) calculated in the free Bose theory. The proof of this equivalence is given in Appendix B. In Appendix $\mathrm{C}$ we demonstrate that the correspondence holds for the commutation relations as well. Readers untroubled by operator manipulations in bosonization can ignore them.

Using Eq. (68) we can then fermionize the $\chi \mathrm{SG}$ Hamiltonian (65) to

$$
\mathcal{H}_{\chi S G}^{\hat{\beta}^{2}=4}=\int_{-L / 2}^{L / 2} d x:\left[-i v_{\mathrm{n}} \psi^{\dagger} \partial_{x} \psi-i \frac{\lambda}{2 \pi}\left(\psi^{\dagger} \partial_{x} \psi^{\dagger}+\psi \partial_{x} \psi\right)\right]: .
$$

The effect of the tunneling term is made transparent by writing the Dirac fermion in terms of its Majorana components: $\psi(x) \equiv\left[\chi_{1}(x)+i \chi_{2}(x)\right] / \sqrt{2}$, where $\chi_{i}^{\dagger}=\chi_{i}$,

$$
\mathcal{H}_{\chi S G}^{\hat{\beta}^{2}=4}=-\frac{1}{2} \int_{-L / 2}^{L / 2} d x:\left[i\left(v_{\mathrm{n}}+\frac{\lambda}{\pi}\right) \chi_{1} \partial_{x} \chi_{1}+i\left(v_{\mathrm{n}}-\frac{\lambda}{\pi}\right) \chi_{2} \partial_{x} \chi_{2}\right]: .
$$

An alternative derivation of this form of the Hamiltonian is given in Appendix D. We see that the effect of tunneling is to split the neutral mode into two Majorana fields with different velocities. This is a $c=1=(1 / 2)+(1 / 2)$ analog of the usual spin-charge separation in one dimension $[c=2=(1)+(1)]$. Since the Hamiltonian (69) is quadratic, modes of the fermion field with momentum $|k| \geq \Lambda$, for some $\Lambda$, do not couple to modes with momentum $|k|<\Lambda$, and thus the tree-level RG flow is exact. Therefore if we define our RG transformation so that the $\lambda=0$ Hamiltonian is a fixed point, we immediately see that the coupling $\lambda$ is exactly marginal, and we have a line of fixed points as asserted at the end of Section III.

Armed with a quadratic representation of the Hamiltonian we can readily compute the partition function and correlation functions exactly. From the expression for the partition function (23) at $\lambda=0$ we see that the characters of the neutral mode that appear at the $\hat{\beta}^{2}=4$ point are $\chi_{1 / 2}^{(4,0)}$ and $\chi_{1 / 2}^{(4,2)}$. These can be written in terms of the characters of the highest weight $h$ representation of the Virasoro algebra with central charge $c$, denoted $\mathcal{V}_{h}^{c}$ :

$$
\chi_{1 / 2}^{(4,0)}(q)=\left[\mathcal{V}_{0}^{1 / 2}(q)\right]^{2}+\left[\mathcal{V}_{1 / 2}^{1 / 2}(q)\right]^{2}, \quad \chi_{1 / 2}^{(4,2)}(q)=2 \mathcal{V}_{0}^{1 / 2}(q) \mathcal{V}_{1 / 2}^{1 / 2}(q)
$$


Since a single chiral Majorana fermion with antiperiodic boundary conditions has a character $\mathcal{V}_{0}^{1 / 2}+\mathcal{V}_{1 / 2}^{1 / 2}$, we see from Eqns. (23), (70), and (71), that the partition function of the $\chi \mathrm{SG}$ theory in isolation is

$$
Z_{\chi S G}^{\hat{\beta}^{2}=4}(\beta)=\left[\mathcal{V}_{0}^{1 / 2}\left(q_{+}\right)+\mathcal{V}_{1 / 2}^{1 / 2}\left(q_{+}\right)\right]\left[\mathcal{V}_{0}^{1 / 2}\left(q_{-}\right)+\mathcal{V}_{1 / 2}^{1 / 2}\left(q_{-}\right)\right]
$$

where $q_{ \pm} \equiv e^{-2 \pi \beta v_{ \pm} / L}$, and $v_{ \pm} \equiv v_{\mathrm{n}} \pm \lambda / \pi$, while the partition function of the full edge theory with tunneling, including the charged mode with the appropriate gluing condition, is

$$
\begin{aligned}
Z^{\hat{\beta}^{2}=4}(\beta)= & \chi_{R_{\mathrm{c}}}^{(2(m+n), 0)}\left(q_{\mathrm{c}}\right)\left[\mathcal{V}_{0}^{1 / 2}\left(q_{+}\right) \mathcal{V}_{0}^{1 / 2}\left(q_{-}\right)+\mathcal{V}_{1 / 2}^{1 / 2}\left(q_{+}\right) \mathcal{V}_{1 / 2}^{1 / 2}\left(q_{-}\right)\right] \\
& +\chi_{R_{\mathrm{c}}}^{(2(m+n), m+n)}\left(q_{\mathrm{c}}\right)\left[\mathcal{V}_{0}^{1 / 2}\left(q_{+}\right) \mathcal{V}_{1 / 2}^{1 / 2}\left(q_{-}\right)+\mathcal{V}_{1 / 2}^{1 / 2}\left(q_{+}\right) \mathcal{V}_{0}^{1 / 2}\left(q_{-}\right)\right]
\end{aligned}
$$

Recall that there are three irreducible Verma modules in the $c=1 / 2$ minimal model with highest weights $h=0,1 / 2$, and $1 / 16$, which in the Ising model terminology correspond to the identity $(I)$, energy $(\epsilon)$ and spin $(\sigma)$ fields. The characters of the first two of these representations appear in the above partition function, the remaining one occurs if one considers the other sector of the neutral mode which involves the characters $\chi_{1 / 2}^{(4,1)}$ and $\chi_{1 / 2}^{(4,3)}$, and which corresponds to the addition of a quasiparticle. These combine to give $\chi_{1 / 2}^{(4,1)}+\chi_{1 / 2}^{(4,3)}=2\left(\mathcal{V}_{1 / 16}^{1 / 2}\right)^{2}$.

Turning now to correlation functions, we can use the transformation (15), and the fermionization of the neutral boson to write the electron (25), density (11), and tunneling (54) operators in terms of the fields $\phi_{\mathrm{c}}$ and $\psi$,

$$
\begin{aligned}
: \Psi_{i}: & =\frac{1}{(2 \pi a)^{(m-1) / 2}} e^{i \sqrt{m-1} \phi_{\mathrm{c}}}\left(\delta_{i 1} \psi^{\dagger}+\delta_{i 2} \psi\right), \\
\rho_{i} & =\frac{1}{4 \pi \sqrt{m-1}} \partial_{x} \phi_{\mathrm{c}}+\frac{(-1)^{i}}{2}: \psi^{\dagger} \psi:, \\
\Theta(x) & =-\frac{i}{2 \pi}:\left(\psi^{\dagger} \partial_{x} \psi^{\dagger}+\psi \partial_{x} \psi\right):,
\end{aligned}
$$

where $i=1,2$ is the layer index. The single-electron Green's function is

$$
-\mathcal{G}_{i j}(\tau, x)=\frac{1}{2\left[2 \pi\left(v_{\mathrm{c}} \tau-i x\right)\right]^{m-1}}\left[\frac{1}{2 \pi\left(v_{+} \tau-i x\right)}+\frac{(-1)^{i+j}}{2 \pi\left(v_{-} \tau-i x\right)}\right],
$$

and we see that in the presence of tunneling an electron has broken up into three pieces, propagating with three different velocities. The two-point functions of the density and tunneling operators are:

$$
\begin{aligned}
\mathcal{D}_{i j}(\tau, x) & =\frac{1}{4(m-1)} \frac{1}{\left[2 \pi\left(v_{\mathrm{c}} \tau-i x\right)\right]^{2}}+\frac{(-1)^{i+j}}{4} \frac{1}{2 \pi\left(v_{+} \tau-i x\right) 2 \pi\left(v_{-} \tau-i x\right)}, \\
\mathcal{T}(\tau, x) & =\frac{1}{\left[2 \pi\left(v_{+} \tau-i x\right)\right]^{4}}+\frac{1}{\left[2 \pi\left(v_{-} \tau-i x\right)\right]^{4}} .
\end{aligned}
$$




\section{FERMIONIZATIONS OF THE CHIRAL SINE-GORDON THEORY}

In this section we describe a general method for fermionizing the $\chi \mathrm{SG}$ theory for $\hat{\beta}^{2} \in \mathbb{Z}^{+}$ by the introduction of auxiliary degrees of freedom. The procedure is illustrated in detail for the cases $\hat{\beta}^{2}=2$ and $\hat{\beta}^{2}=4$. We also show that for these two points the fermionized theory with auxiliary degrees of freedom can also be solved exactly, and the projection onto the $\chi \mathrm{SG}$ Hilbert space can be performed explicitly, reproducing the results of the exact solutions given above. In terms of the solution of the double-layer problem, the method described here is redundant. However, we believe it may be useful in the study of quantum Hall systems with more than two layers. This section can be skipped since the subsequent sections are independent of the material presented here.

\section{A. General Strategy}

In the case of the standard SG theory it is possible to fermionize the theory at all values of $\hat{\beta}^{2} \leq 4$ to the massive Thirring model [20]. For the $\chi \mathrm{SG}$ theory we have found fermionizations at the discrete points $\hat{\beta}^{2} \in \mathbb{Z}^{+}$. Recall that all the points in the bosonic sequence $\left(\hat{\beta}^{2} \in \mathbb{Z}_{\text {even }}^{+}\right)$correspond to some double-layer quantum Hall system, while none of the points in the fermionic sequence $\left(\hat{\beta}^{2} \in \mathbb{Z}_{\text {odd }}^{+}\right)$do. The scaling dimension of the tunneling perturbation is an integer in the bosonic sequence and a half-integer in the fermionic one. We defer discussion of the fermionic sequence until Section VI.

The algebraic identity at the heart of fermionization (or bosonization) is the Cauchy

formula for the determinant of an $N \times N$ matrix $M_{i j}^{(N)} \equiv 1 /\left(z_{i}-z_{j}^{\prime}\right)$ which is a function of $2 N$ complex variables $z_{i}, z_{i}^{\prime}, i=1, \ldots, N$ [25]:

$$
(-1)^{N(N-1) / 2} \operatorname{det} M^{(N)}=\frac{\prod_{1 \leq i<j \leq N}\left(z_{i}-z_{j}\right)\left(z_{i}^{\prime}-z_{j}^{\prime}\right)}{\prod_{1 \leq i, j \leq N}\left(z_{i}-z_{j}^{\prime}\right)} .
$$

The determinant on the l.h.s. is proportional to the $2 N$-point correlation function of a single chiral Dirac fermion:

$$
\left\langle\prod_{i=1}^{N} \psi\left(\tau_{i}, x_{i}\right) \prod_{i=1}^{N} \psi^{\dagger}\left(\tau_{i}^{\prime}, x_{i}^{\prime}\right)\right\rangle=\frac{(-1)^{N(N-1) / 2}}{(2 \pi)^{N}} \operatorname{det} M^{(N)},
$$

where $z_{i} \equiv\left(v \tau_{i}-i x_{i}\right)$, and similarly for the primed coordinates, $v$ is the velocity of the fermion, and time ordering will be implicit from here on. We desire a fermionic representation of the tunneling operator, Eq. (54). The $2 N$-point correlation function of the tunneling operator is a linear combination of terms of the form

$$
\frac{1}{a^{N \hat{\beta}^{2}}}\left\langle\prod_{i=1}^{N} e^{i \hat{\beta} \phi\left(\tau_{i}, x_{i}\right)} \prod_{i=1}^{N} e^{-i \hat{\beta} \phi\left(\tau_{i}^{\prime}, x_{i}^{\prime}\right)}\right\rangle=\left[\frac{\prod_{1 \leq i<j \leq N}\left(z_{i}-z_{j}\right)\left(z_{i}^{\prime}-z_{j}^{\prime}\right)}{\prod_{1 \leq i, j \leq N}\left(z_{i}-z_{j}^{\prime}\right)}\right]^{\hat{\beta}^{2}}=\left[\operatorname{det} M^{(N)}\right]^{\hat{\beta}^{2}}
$$

For the bosonic sequence it is clear from Eq. (81) that the $\hat{\beta}^{2}$ power of the determinant can be reproduced by an identification of the form 


$$
e^{i \hat{\beta} \phi(x)} \sim \prod_{i=1}^{\hat{\beta}^{2} / 2} \psi_{i}(x) \prod_{j=\hat{\beta}^{2} / 2+1}^{\hat{\beta}^{2}} \psi_{j}^{\dagger}(x)
$$

where the $\psi_{i}(x)$ are $\hat{\beta}^{2}$ independent chiral Dirac fermions with identical velocities. This construction closely resembles Jain's bulk parton construction for hierarchical fractional quantum Hall states [30] and its edge analog [12], although the motivation here, a desire for a fermionic representation of the tunneling operator, is very different.

In the $\chi \mathrm{SG}$ Hamiltonian there is only a single chiral boson (with central charge $c=1$ ), so we first have to add $\left(\hat{\beta}^{2}-1\right)$ auxiliary $c=1$ degrees of freedom (e.g., chiral bosons) in order to carry out this procedure. Of the points in the bosonic sequence, only the first two $\left(\hat{\beta}^{2}=2,4\right)$ occur in the region where the cosine term is not irrelevant [by Eq. (38)], and these are the ones we now describe in detail.

\section{B. Relevant Tunneling, $\hat{\beta}^{2}=2$}

As noted above, an additional degree of freedom is necessary for a straightforward fermionization of the $\chi \mathrm{SG}$ Hamiltonian, so we add to the Hamiltonian an auxiliary free compact chiral boson $(\hat{\phi})$ with radius $\hat{R}$, topological charge $\hat{N}$, and a velocity equal to the velocity of $\phi$. The full Hamiltonian is then:

$$
\begin{aligned}
\hat{\mathcal{H}}_{\chi S G}^{\hat{\beta}^{2}=2} & \equiv \mathcal{H}_{\chi S G}^{\hat{\beta}^{2}=2}[\phi]+\mathcal{H}_{F}[\hat{\phi}] \\
& =\int_{-L / 2}^{L / 2} d x\left[\frac{1}{4 \pi} v_{\mathrm{n}}:\left(\partial_{x} \phi\right)^{2}:+\frac{1}{4 \pi} v_{\mathrm{n}}:\left(\partial_{x} \hat{\phi}\right)^{2}:+\frac{\lambda}{2 \pi a}\left(e^{i \sqrt{2} \phi}+\text { h.c. }\right)\right] .
\end{aligned}
$$

We then perform a canonical transformation which mixes the field appearing in the tunneling term with the auxiliary boson

$$
\left(\begin{array}{c}
\hat{\phi} \\
\phi
\end{array}\right)=\frac{1}{\sqrt{2}}\left(\begin{array}{cc}
1 & 1 \\
1 & -1
\end{array}\right)\left(\begin{array}{l}
\theta_{1} \\
\theta_{2}
\end{array}\right)
$$

in terms of which we find

$$
\hat{\mathcal{H}}_{\chi S G}^{\hat{\beta}^{2}=2}=\int_{-L / 2}^{L / 2} d x\left\{\frac{1}{4 \pi} v_{\mathrm{n}}: \partial_{x} \theta_{i} \partial_{x} \theta_{i}:+\frac{\lambda}{2 \pi a}\left[e^{i\left(\theta_{1}-\theta_{2}\right)}+\text { h.c. }\right]\right\} .
$$

The topological charge dependent part of the transformation in Eq. (85) is

$$
\hat{R} \hat{N}=\frac{1}{\sqrt{2}}\left(N_{1}^{\theta} R_{1}^{\theta}+N_{2}^{\theta} R_{2}^{\theta}\right), \quad \frac{1}{\sqrt{2}} N_{\mathrm{n}}=\frac{1}{\sqrt{2}}\left(N_{1}^{\theta} R_{1}^{\theta}-N_{2}^{\theta} R_{2}^{\theta}\right),
$$

where $N_{i}^{\theta}, R_{i}^{\theta}$ are the topological charge and radius of the field $\theta_{i}(x)$, respectively. We choose $\hat{R}=1 / \sqrt{2}$ so that the requirement $N_{i}^{\theta} \in \mathbb{Z}$ is consistent with setting $R_{i}^{\theta}=1$. These choices also impose the gluing condition $\hat{N}+N_{\mathrm{n}} \in \mathbb{Z}_{\text {even }}$. With these values of the radii for the $\theta_{i}$ fields, we can define two chiral fermion operators

$$
\psi_{i}(x)=\frac{1}{\sqrt{2 \pi a}} e^{i \theta_{i}(x)}
$$


These two flavors of fermions commute, but once again this can be fixed without modifying what follows, and the details are given in Appendix A. Using this definition in Eq. (86) we have a fermionized Hamiltonian

$$
\hat{\mathcal{H}}_{\chi S G}^{\hat{\beta}^{2}=2}=\int_{-L / 2}^{L / 2} d x:\left[-i v_{\mathrm{n}} \psi_{i}^{\dagger} \partial_{x} \psi_{i}-\lambda\left(\psi_{2}^{\dagger} \psi_{1}+\text { h.c. }\right)\right]: \text {. }
$$

As noted in Section [1], the fermion fields introduced in Eq. (88) obey antiperiodic boundary conditions and therefore the allowed values of the momentum $k$ for the Fourier mode operators $c_{k i}$ of $\psi_{i}(x)$ are $k \in(2 \pi / L)(\mathbb{Z}+1 / 2)$. The fermionic normal ordering introduced in Eq. (89) is defined with respect to the (non-degenerate) ground state of this Hamiltonian at $\lambda=0$ which is annihilated by $c_{k i}$ for $k>0$ and $c_{k i}^{\dagger}$ for $k<0$. By introducing $\psi_{ \pm} \equiv\left(\psi_{1} \pm \psi_{2}\right) / \sqrt{2}$ the Hamiltonian is diagonalized:

$$
\hat{\mathcal{H}}_{\chi S G}^{\hat{\beta}^{2}=2}=\int_{-L / 2}^{L / 2} d x:\left[-i v_{\mathrm{n}}\left(\psi_{+}^{\dagger} \partial_{x} \psi_{+}+\psi_{-}^{\dagger} \partial_{x} \psi_{-}\right)-\lambda\left(\psi_{+}^{\dagger} \psi_{+}+\psi_{-}^{\dagger} \psi_{-}\right)\right]: .
$$

Note that in the case of the 110 state the charged boson $\left(\phi_{\mathrm{c}}\right)$ has radius $R_{\mathrm{c}}=1 / \sqrt{2}$ and hence the fermionization can be accomplished without adding an auxiliary degree of freedom by replacing $\hat{\phi}$ by $\phi_{\mathrm{c}}$ in Eq. (85). The result of this procedure is nothing more than the chiral fermion description of the original Hamiltonian of two uncorrelated, interacting, $\nu=1$ edges, given above in Eq. (61). For the 110 state the advantage of using an auxiliary boson rather than the charged mode in the fermionization is that in the former case the theory is quadratic (90), whereas in the latter case it is not (61).

Having solved the theory with the auxiliary boson, we now show at the level of the partition function that projecting out the unphysical degree of freedom from the spectrum of Eq. (90) reproduces the result from the direct solution in Section IV. From Eq. (90) the partition functions of the $\chi \mathrm{SG}$ theory with the auxiliary boson is

$$
\hat{Z}_{\chi S G}^{\hat{\beta}^{2}=2}(\beta)=\frac{1}{\varphi\left(q_{\mathrm{n}}\right)^{2}} \sum_{N_{+}, N_{-} \in \mathbb{Z}} e^{-\beta \lambda\left(N_{+}+N_{-}\right)} q_{\mathrm{n}}^{\frac{1}{2}\left(N_{+}^{2}+N_{-}^{2}\right)} .
$$

To write the partition function including the charged mode we must again consider gluing conditions. We must separate states according to their $N_{\mathrm{n}}$-parity, which because of the condition $\hat{N}+N_{\mathrm{n}} \in \mathbb{Z}_{\text {even }}$, is equivalent in their $\hat{N}$-parity. Since $\hat{N}$ is just the total fermion number measured with respect to the $\lambda=0$ ground state, we see the partition function of the full edge theory is:

$$
\hat{Z}^{\hat{\beta}^{2}=2}(\beta)=\chi_{R_{\mathrm{c}}}^{(2(m+n), 0)}\left(q_{\mathrm{c}}\right) \hat{Z}_{\chi S G}^{\hat{\beta}^{2}=2, \text { even }}+\chi_{R_{\mathrm{c}}}^{(2(m+n), m+n)}\left(q_{\mathrm{c}}\right) \hat{Z}_{\chi S G}^{\hat{\beta}^{2}=2, \text { odd }},
$$

where $\hat{Z}_{\chi S G}^{\hat{\beta}^{2}=2, \text { even }}$ is given by Eq. (91) with the restriction $N_{+}+N_{-} \in \mathbb{Z}_{\text {even }}$ and similarly for $\hat{Z}_{\chi S G}^{\hat{\beta}^{2}=2 \text {, odd }}$. To project out the radius $R=1 / \sqrt{2}$ auxiliary boson we added $(\hat{\phi})$ in Eq. (84) we must evaluate

$$
Z_{\chi S G}^{\hat{\beta}^{2}=2}(\beta)=\frac{\hat{Z}_{\chi S G}^{\hat{\beta}^{2}=2, \text { even }}}{\chi_{1 / \sqrt{2}}^{(2,0)}\left(q_{\mathrm{n}}\right)}+\frac{\hat{Z}_{\chi S G}^{\hat{\beta}^{2}=2, \text { odd }}}{\chi_{1 / \sqrt{2}}^{(2,1)}\left(q_{\mathrm{n}}\right)}
$$


for the $\chi \mathrm{SG}$ theory in isolation and

$$
Z^{\hat{\beta}^{2}=2}(\beta)=\chi_{R_{\mathrm{c}}}^{(2(m+n), 0)}\left(q_{\mathrm{c}}\right) \frac{\hat{Z}_{\chi S G}^{\hat{\beta}^{2}=2, \text { even }}}{\chi_{1 / \sqrt{2}}^{(2,0)}\left(q_{\mathrm{n}}\right)}+\chi_{R_{\mathrm{c}}}^{(2(m+n), m+n)}\left(q_{\mathrm{c}}\right) \frac{\hat{Z}_{\chi S G}^{\hat{\beta}^{2}=2, \text { odd }}}{\chi_{1 / \sqrt{2}}^{(2,1)}\left(q_{\mathrm{n}}\right)},
$$

for the full edge theory. It is useful at this point to recall the definitions of the Jacobi theta functions:

$$
\begin{aligned}
\Theta_{2}(\nu \mid \tau) & =\sum_{r \in \mathbb{Z}+\frac{1}{2}} e^{2 \pi i \nu r} e^{i \pi \tau r^{2}}, \\
\Theta_{3}(\nu \mid \tau) & =\sum_{r \in \mathbb{Z}} e^{2 \pi i \nu r} e^{i \pi \tau r^{2}}, \\
\Theta_{4}(\nu \mid \tau) & =\sum_{r \in \mathbb{Z}}(-1)^{r} e^{2 \pi i \nu r} e^{i \pi \tau r^{2}} .
\end{aligned}
$$

If we define $\bar{\nu} \equiv i \beta \lambda / 2 \pi$ and $\bar{\tau} \equiv i \beta v_{\mathrm{n}} / L$, we see from the definition of the chiral boson character (24) and Eqns. (95) that

$$
\chi_{1 / \sqrt{2}}^{(2,0)}\left(q_{\mathrm{n}}\right)=\frac{1}{\varphi\left(q_{\mathrm{n}}\right)} \Theta_{3}(0 \mid 2 \bar{\tau}), \quad \chi_{1 / \sqrt{2}}^{(2,1)}\left(q_{\mathrm{n}}\right)=\frac{1}{\varphi\left(q_{\mathrm{n}}\right)} \Theta_{2}(0 \mid 2 \bar{\tau}) .
$$

Next note that we can rewrite the sums appearing in $\hat{Z}_{\chi S G}^{\hat{\beta}^{2}=2 \text {, even }}$ and $\hat{Z}_{\chi S G}^{\hat{\beta}^{2}=2 \text {, odd }}$ using

$$
\begin{aligned}
\sum_{N_{+}+N_{-} \in \mathbb{Z}_{\text {even }}} & =\sum_{N_{+}, N_{-} \in \mathbb{Z}} \frac{1}{2}\left[1+(-1)^{N_{+}+N_{-}}\right], \\
\sum_{N_{+}+N_{-} \in \mathbb{Z}_{\text {odd }}} & =\sum_{N_{+}, N_{-} \in \mathbb{Z}} \frac{1}{2}\left[1-(-1)^{N_{+}+N_{-}}\right],
\end{aligned}
$$

and hence

$$
\begin{aligned}
\hat{Z}_{\chi S G}^{\hat{\beta}^{2}=2, \text { even }} & =\frac{1}{2 \varphi\left(q_{\mathrm{n}}\right)^{2}}\left\{\left[\Theta_{3}(\bar{\nu} \mid \bar{\tau})\right]^{2}+\left[\Theta_{4}(\bar{\nu} \mid \bar{\tau})\right]^{2}\right\} \\
\hat{Z}_{\chi S G}^{\hat{\beta}^{2}=2, \text { odd }} & =\frac{1}{2 \varphi\left(q_{\mathrm{n}}\right)^{2}}\left\{\left[\Theta_{3}(\bar{\nu} \mid \bar{\tau})\right]^{2}-\left[\Theta_{4}(\bar{\nu} \mid \bar{\tau})\right]^{2}\right\} .
\end{aligned}
$$

Finally, using the standard doubling identities [31]

$$
\begin{aligned}
& \Theta_{3}(2 \nu \mid 2 \tau)=\frac{\left[\Theta_{3}(\nu \mid \tau)\right]^{2}+\left[\Theta_{4}(\nu \mid \tau)\right]^{2}}{2 \Theta_{3}(0 \mid 2 \tau)}, \\
& \Theta_{2}(2 \nu \mid 2 \tau)=\frac{\left[\Theta_{3}(\nu \mid \tau)\right]^{2}-\left[\Theta_{4}(\nu \mid \tau)\right]^{2}}{2 \Theta_{2}(0 \mid 2 \tau)},
\end{aligned}
$$

and Eqns. (96) and (98) in (93) and (94) we obtain

$$
\begin{aligned}
& Z_{\chi S G}^{\hat{\beta}^{2}=2}(\beta)=\frac{1}{\varphi\left(q_{\mathrm{n}}\right)}\left[\Theta_{3}(2 \bar{\nu} \mid 2 \bar{\tau})+\Theta_{2}(2 \bar{\nu} \mid 2 \bar{\tau})\right] \\
& Z^{\hat{\beta}^{2}=2}(\beta)=\chi_{R_{\mathrm{c}}}^{(2(m+n), 0)}\left(q_{\mathrm{c}}\right) \frac{1}{\varphi\left(q_{\mathrm{n}}\right)} \Theta_{3}(2 \bar{\nu} \mid 2 \bar{\tau})+\chi_{R_{\mathrm{c}}}^{(2(m+n), m+n)}\left(q_{\mathrm{c}}\right) \frac{1}{\varphi\left(q_{\mathrm{n}}\right)} \Theta_{2}(2 \bar{\nu} \mid 2 \bar{\tau}),
\end{aligned}
$$


which by Eq. (95) are in precise agreement with the results of the exact solution in the previous section, Eqns. (52) and (53). We have also checked that correlation functions calculated with the fermionic Hamiltonian (90) are identical to those found in Eqns. (57) and (58).

\section{Marginal Tunneling, $\hat{\beta}^{2}=4$}

We next consider the generic multi-flavor fermionization of the $\chi \mathrm{SG}$ Hamiltonian at $\hat{\beta}^{2}=4$. We add to $\mathcal{H}_{\chi S G}^{\hat{\beta}^{2}=4}$ the Hamiltonian for three free chiral bosons $\left(\hat{\phi}_{i}\right)$ with radii $\hat{R}_{i}$ and topological charges $\hat{N}_{i}$ :

$$
\begin{aligned}
\hat{\mathcal{H}}_{\chi S G}^{\hat{\beta}^{2}=4} \equiv \mathcal{H}_{\chi S G}^{\hat{\beta}^{2}=4}[\phi] & +\mathcal{H}_{F}\left[\hat{\phi}_{1}, \hat{\phi}_{2}, \hat{\phi}_{3}\right] \\
=\int_{-L / 2}^{L / 2} d x & \left\{\frac{1}{4 \pi}:\left[v_{\mathrm{n}}\left(\partial_{x} \phi\right)^{2}+\left(v_{\mathrm{n}}+\frac{\lambda}{2 \pi}\right)\left[\left(\partial_{x} \hat{\phi}_{1}\right)^{2}+\left(\partial_{x} \hat{\phi}_{2}\right)^{2}\right]\right.\right. \\
& \left.\left.+\left(v_{\mathrm{n}}+\frac{\lambda}{\pi}\right)\left(\partial_{x} \hat{\phi}_{3}\right)^{2}\right]:+\frac{2 \lambda}{(2 \pi a)^{2}} \cos [2 \phi(x)]\right\} .
\end{aligned}
$$

The velocities of the auxiliary fields are completely at our discretion, and the motivation for the specific choice made here will become clear below. We perform an orthogonal (canonical) transformation which mixes the physical mode $(\phi)$ with the auxiliary bosons:

$$
\left(\begin{array}{c}
\phi \\
\hat{\phi}_{1} \\
\hat{\phi}_{2} \\
\hat{\phi}_{3}
\end{array}\right)=\frac{1}{2}\left(\begin{array}{cccc}
1 & -1 & 1 & -1 \\
\sqrt{2} & \sqrt{2} & 0 & 0 \\
0 & 0 & \sqrt{2} & \sqrt{2} \\
1 & -1 & -1 & 1
\end{array}\right)\left(\begin{array}{c}
\theta_{1} \\
\theta_{2} \\
\theta_{3} \\
\theta_{4}
\end{array}\right) .
$$

In terms of these new bosons our Hamiltonian is:

$$
\begin{aligned}
\hat{\mathcal{H}}_{\chi S G}^{\hat{\beta}^{2}=4}= & \int_{-L / 2}^{L / 2} d x\left\{\frac{1}{4 \pi}\left(v_{\mathrm{n}}+\frac{\lambda}{2 \pi}\right): \partial_{x} \theta_{i} \partial_{x} \theta_{i}:+\frac{\lambda}{(2 \pi a)^{2}}\left[e^{i\left(\theta_{1}-\theta_{2}+\theta_{3}-\theta_{4}\right)}+\text { h.c. }\right]\right. \\
& \left.+\frac{\lambda}{2 \pi}:\left(\partial_{x} \theta_{1}-\partial_{x} \theta_{2}\right)\left(\partial_{x} \theta_{4}-\partial_{x} \theta_{3}\right):\right\} .
\end{aligned}
$$

The topological charge dependent part of the transformation in Eq. (102) is

$$
\begin{array}{ll}
\frac{1}{2} N_{\mathrm{n}}=\frac{1}{2}\left(R_{1}^{\theta} N_{1}^{\theta}-R_{2}^{\theta} N_{2}^{\theta}+R_{3}^{\theta} N_{3}^{\theta}-R_{4}^{\theta} N_{4}^{\theta}\right), & \hat{R}_{1} \hat{N}_{1}=\frac{1}{\sqrt{2}}\left(R_{1}^{\theta} N_{1}^{\theta}+R_{2}^{\theta} N_{2}^{\theta}\right), \\
\hat{R}_{3} \hat{N}_{3}=\frac{1}{2}\left(R_{1}^{\theta} N_{1}^{\theta}-R_{2}^{\theta} N_{2}^{\theta}-R_{3}^{\theta} N_{3}^{\theta}+R_{4}^{\theta} N_{4}^{\theta}\right), & \hat{R}_{2} \hat{N}_{2}=\frac{1}{\sqrt{2}}\left(R_{3}^{\theta} N_{3}^{\theta}+R_{4}^{\theta} N_{4}^{\theta}\right),
\end{array}
$$

where $N_{i}^{\theta}, R_{i}^{\theta}$ are the topological charge and radius of the field $\theta_{i}(x)$, respectively. We again want to use our freedom to choose the quantities $\hat{R}_{i}$ to ensure $R_{i}^{\theta}=1$. Clearly $\hat{R}_{1}=\hat{R}_{2}=1 / \sqrt{2}, \hat{R}_{3}=1 / 2$ accomplishes this. Since the $\theta_{i}$ fields have unit radius we can introduce a quartet of chiral fermion operators

$$
\psi_{i}(x)=\frac{1}{\sqrt{2 \pi a}} e^{i \theta_{i}(x)} .
$$


Once again these fermion operators are defined here without the Klein factors necessary to ensure the proper anticommutation relations. The fact that this can be remedied without modifying the form of the Hamiltonian is demonstrated in Appendix A.

In terms of these fermions Eq. (103) reads:

$$
\begin{aligned}
\hat{\mathcal{H}}_{\chi S G}^{\hat{\beta}^{2}=4}= & \int_{-L / 2}^{L / 2} d x:\left\{-i v_{\mathrm{n}}^{\prime} \psi_{i}^{\dagger} \partial_{x} \psi_{i}+\frac{\lambda^{\prime}}{2}\left[\psi_{1} \psi_{2}^{\dagger} \psi_{3} \psi_{4}^{\dagger}+\psi_{4} \psi_{3}^{\dagger} \psi_{2} \psi_{1}^{\dagger}\right.\right. \\
& \left.\left.+\frac{1}{2}\left(\psi_{1}^{\dagger} \psi_{1}-\psi_{2}^{\dagger} \psi_{2}\right)\left(\psi_{4}^{\dagger} \psi_{4}-\psi_{3}^{\dagger} \psi_{3}\right)\right]\right\}:
\end{aligned}
$$

where $v_{\mathrm{n}}^{\prime} \equiv v_{\mathrm{n}}+\lambda / 2 \pi$, and $\lambda^{\prime} \equiv 2 \lambda$. The fermion normal ordering in Eq. (106) is again defined with respect to the $\lambda=0$ ground state which satisfies $c_{k i}|\Omega\rangle=c_{-k i}^{\dagger}|\Omega\rangle=0$ for $k>0$ and all $i$, where $k \in(2 \pi / L)(\mathbb{Z}+1 / 2)$ and $c_{k i}$ are the Fourier components of $\psi_{i}(x)$. At this value of $\hat{\beta}^{2}$ the $\lambda=0$ ground state is the exact ground state of the Hamiltonian for $\lambda \neq 0$ (in contrast to the $\hat{\beta}^{2}=2$ case where the $\lambda=0$ ground state is an exact eigenstate for $\lambda \neq 0$, but not the ground state). Assembling our four spinless fermions into a pair of (pseudo-)spin-1/2 fermions according to

$$
\Phi_{1} \equiv\left(\begin{array}{c}
\psi_{1} \\
\psi_{2}
\end{array}\right), \quad \Phi_{2} \equiv\left(\begin{array}{c}
\psi_{4} \\
\psi_{3}
\end{array}\right)
$$

the Hamiltonian (106) can be written as a quadratic form

$$
\hat{\mathcal{H}}_{\chi S G}^{\hat{\beta}^{2}=4}=\int_{-L / 2}^{L / 2} d x:\left[\frac{\pi v_{\mathrm{n}}^{\prime}}{2}\left(J_{1}^{2}+J_{2}^{2}\right)+\frac{2 \pi v_{\mathrm{n}}^{\prime}}{3}\left(\mathbf{J}_{1}^{2}+\mathbf{J}_{2}^{2}\right)+\lambda^{\prime} \mathbf{J}_{1} \cdot \mathbf{J}_{2}\right]:
$$

in terms of the currents

$$
J_{i}(x) \equiv: \Phi_{i \alpha}^{\dagger}(x) \Phi_{i \alpha}(x):, \quad J_{i}^{a}(x) \equiv \frac{1}{2}: \Phi_{i \alpha}^{\dagger}(x) \sigma_{\alpha \beta}^{a} \Phi_{i \beta}(x): .
$$

where $\sigma^{a}, a=x, y, z$, are the Pauli matrices. The reason for our choice of velocities in Eq. (101) is now apparent: along with the transformation in Eq. (102) it produces a combination of four-Fermi couplings in Eq. (106) that can be written as a scalar product of currents. This is not a unique construction, we can obtain the same final form (108) with a different choice of velocities by modifying the transformation (102).

Defining the Fourier components of the currents

$$
J_{i, n} \equiv \int_{-L / 2}^{L / 2} d x J_{i}(x) e^{-2 \pi i n x / L}, \quad \mathbf{J}_{i, n} \equiv \int_{-L / 2}^{L / 2} d x \mathbf{J}_{i}(x) e^{-2 \pi i n x / L}, \quad n \in \mathbb{Z},
$$

the algebra is

$$
\begin{aligned}
& {\left[J_{i, n}, J_{j, m}\right]=2 n \delta_{i j} \delta_{n+m, 0}, \quad\left[J_{i, n}, J_{j, m}^{a}\right]=0,} \\
& {\left[J_{i, n}^{a}, J_{j, m}^{b}\right]=\frac{n}{2} \delta_{i j} \delta^{a b} \delta_{n+m, 0}+i \delta_{i j} \epsilon^{a b c} J_{i, n+m}^{c} .}
\end{aligned}
$$

We therefore have two $\hat{u}(1)\left(J_{i}\right)$ and two $\widehat{s u}(2)_{1}\left(\mathbf{J}_{i}\right)$ Kac-Moody algebras. From Eqns. (109) and (110) we find that the ground state satisfies 


$$
J_{i, n}|\Omega\rangle=J_{i, n}^{a}|\Omega\rangle=0, \text { for } \quad i=1,2 ; \quad a=x, y, z ; \quad n \geq 0,
$$

and therefore normal ordering for the current operator modes is defined by moving any current with momentum index $n \leq 0$ to the right past any current with index $m>0$ with which it has a non-trivial commutator.

To solve Eq. (108), the spin part of which is the Sugawara form of two coupled $\widehat{s u}(2)_{1}$ Wess-Zumino-Witten models [25], we first note that we can define:

$$
\mathcal{L}_{n}^{(i)} \equiv \frac{1}{2}: J_{i, m} J_{i, n-m}: \quad L_{n}^{(i)} \equiv \frac{1}{3}: J_{i, m}^{a} J_{i, n-m}^{a}:
$$

These four sets of operators obey four independent $c=1$ Virasoro algebras. The operators $\mathbf{J}_{+} \equiv \mathbf{J}_{1}+\mathbf{J}_{2}$ obey an $\widehat{s u}(2)_{2}$ diagonal subalgebra of $\widehat{s u}(2)_{1} \oplus \widehat{s u}(2)_{1}$, and the bilinear operators formed from them

$$
L_{n}^{(+)} \equiv \frac{1}{4}: J_{+, m}^{a} J_{+, n-m}^{a}:
$$

obey a $c=3 / 2$ Virasoro algebra. From the GKO coset construction, the operators $L_{n}^{(1 / 2)} \equiv$ $L_{n}^{(1)}+L_{n}^{(2)}-L_{n}^{(+)}$form a $c=1 / 2$ Virasoro algebra which is independent of the one formed from the $\mathbf{J}_{+, n}$ currents, i.e., $\left[L_{n}^{(1 / 2)}, L_{m}^{(+)}\right]=0$. Using these observations and the trivial identity

$$
: \mathbf{J}_{1}(x) \cdot \mathbf{J}_{2}(x):=\frac{1}{2} \frac{:\left[\mathbf{J}_{+}(x)\right]^{2}:}{4}-\frac{3}{2}\left\{\frac{:\left[\mathbf{J}_{1}(x)\right]^{2}:+:\left[\mathbf{J}_{2}(x)\right]^{2}:}{3}-\frac{:\left[\mathbf{J}_{+}(x)\right]^{2}:}{4}\right\},
$$

the Hamiltonian becomes

$$
\hat{\mathcal{H}}_{\chi S G}^{\hat{\beta}^{2}=4}=\frac{\pi v_{\mathrm{n}}^{\prime}}{L}\left[\mathcal{L}_{0}^{(1)}+\mathcal{L}_{0}^{(2)}\right]+\left[\frac{2 \pi v_{\mathrm{n}}^{\prime}}{L}+\frac{\lambda^{\prime}}{2 L}\right] L_{0}^{(+)}+\left[\frac{2 \pi v_{\mathrm{n}}^{\prime}}{L}-\frac{3 \lambda^{\prime}}{2 L}\right] L_{0}^{(1 / 2)} .
$$

By transforming from $L_{n}^{(1)}, L_{n}^{(2)}$ to $L_{n}^{(+)}, L_{n}^{(1 / 2)}$ we have succeeded in diagonalizing the Hamiltonian. From Eqns. (112), (113), (114), and the definition of $L_{n}^{(1 / 2)}$ one finds that the ground state $|\Omega\rangle$ is annihilated by $\mathcal{L}_{n}^{(i)}, L_{n}^{(+)}, L_{n}^{(1 / 2)}$ for $i=1,2$ and $n \geq 0$. Excited states are obtained by acting on $|\Omega\rangle$ with combinations of Virasoro generators with $n<0$.

We now project out the auxiliary degrees of freedom we added in Eq. (101). The Hilbert space of the $\chi \mathrm{SG}$ Hamiltonian is the subspace of the Hilbert space of $\hat{\mathcal{H}}_{\chi S G}^{\hat{\beta}^{2}=4}$ for which the auxiliary chiral bosons are in their ground state. We define a state $|\gamma\rangle$ as physical (i.e., belonging to the Hilbert space of the $\chi$ SG Hamiltonian) if it satisfies

$$
\int_{-L / 2}^{L / 2} d x:\left(\partial_{x} \hat{\phi}_{i}\right)^{2}:|\gamma\rangle=0, \text { for } \quad i=1,2,3
$$

Using Eqns. (102), (105), (107), and (109), these conditions can be written in terms of currents as

$$
\begin{aligned}
& \int_{-L / 2}^{L / 2} d x:\left[J_{i}(x)\right]^{2}:|\gamma\rangle=0 \quad \text { for } \quad i=1,2 \\
& \int_{-L / 2}^{L / 2} d x:\left[J_{+}^{z}(x)\right]^{2}:|\gamma\rangle=0
\end{aligned}
$$


We note that these quadratic conditions can be recast as linear constraints. For example, using Eq. (110) in Eq. (118) and acting on the left with $\langle\gamma|$ we find

$$
\| J_{i, 0}|\gamma\rangle\left\|^{2}+2 \sum_{n=1}^{\infty}\right\| J_{i, n}|\gamma\rangle \|^{2}=0
$$

where we have used $\left(J_{i, n}\right)^{\dagger}=J_{i,-n}$. Since the norm is positive semi-definite we see that Eq. (118) is equivalent to the set of linear conditions $J_{i, n}|\gamma\rangle=0$ for $n \geq 0$. The condition in Eq. (118) states that we should project out the $\widehat{u}(1)$ algebras, i.e., acting on any physical state with $\mathcal{L}_{-n}^{(i)}$ for $i=1,2$, and $n>0$, necessarily produces an unphysical state. From the Hamiltonian (116), and Eq. (118) we have

$$
\hat{\mathcal{H}}_{\chi S G}^{\hat{\beta}^{2}=4}|\gamma\rangle=\left(\frac{2 \pi}{L}\right)\left[\left(v_{\mathrm{n}}^{\prime}+\frac{\lambda^{\prime}}{4 \pi}\right) L_{0}^{(+)}+\left(v_{\mathrm{n}}^{\prime}-\frac{3 \lambda^{\prime}}{4 \pi}\right) L_{0}^{(1 / 2)}\right]|\gamma\rangle,
$$

if $|\gamma\rangle$ is physical. The second projection condition, Eq. (119), is slightly more subtle. We are aided by the observation that the operators $J_{+, n}^{z}$ obey a $\widehat{u}(1)$ subalgebra of the $\widehat{s u}(2)_{2}$ algebra generated by $\mathbf{J}_{+, n}$. Therefore

$$
L_{n}^{(z)} \equiv \frac{1}{2}: J_{+, m}^{z} J_{+, n-m}^{z}:
$$

obey a $c=1$ Virasoro algebra and we can once again use the GKO construction to define operators $L_{n}^{\prime(1 / 2)} \equiv L_{n}^{(+)}-L_{n}^{(z)}$ which are independent of the $L_{n}^{(z)}$ (i.e., $\left.\left[L_{n}^{(1 / 2)}, L_{m}^{(z)}\right]=0\right)$ and which obey a $c=1 / 2$ Virasoro algebra. Using $L_{0}^{(+)}=L_{0}^{\prime(1 / 2)}+L_{0}^{(z)}$ and Eqns. (119), (121) and $(122)$ we arrive at

$$
\hat{\mathcal{H}}_{\chi S G}^{\hat{\beta}^{2}=4}|\gamma\rangle=\mathcal{H}_{\chi S G}^{\hat{\beta}^{2}=4}|\gamma\rangle=\left(\frac{2 \pi}{L}\right)\left[\left(v_{\mathrm{n}}^{\prime}+\frac{\lambda^{\prime}}{4 \pi}\right) L_{0}^{\prime(1 / 2)}+\left(v_{\mathrm{n}}^{\prime}-\frac{3 \lambda^{\prime}}{4 \pi}\right) L_{0}^{(1 / 2)}\right]|\gamma\rangle,
$$

if $|\gamma\rangle$ is a physical state. One can readily show $\left[L_{n}^{\prime(1 / 2)}, L_{m}^{(1 / 2)}\right]=0$ and therefore from Eq. (123) we see that the $\chi \mathrm{SG}$ Hamiltonian can be written as the sum of two independent $c=1 / 2$ pieces with velocities $\left(v_{\mathrm{n}}^{\prime}+\lambda^{\prime} / 4 \pi\right)=v_{\mathrm{n}}+\lambda / \pi=v_{+}$and $\left(v_{\mathrm{n}}^{\prime}-3 \lambda^{\prime} / 4 \pi\right)=v_{\mathrm{n}}-\lambda / \pi=v_{-}$, in precise agreement with the expression (70) obtained by the direct solution in Section [V], since a Majorana fermion has a central charge of $1 / 2$.

\section{FERMIONIC SEQUENCE}

In Section $\mathrm{V}$ we discussed the generic fermionization of the $\chi \mathrm{SG}$ theory for points in the bosonic sequence $\left(\hat{\beta}^{2} \in \mathbb{Z}_{\text {even }}^{+}\right)$, which involves the introduction of $\left(\hat{\beta}^{2}-1\right)$ auxiliary chiral bosons. This procedure can also be applied to the fermionic sequence $\left(\hat{\beta}^{2} \in \mathbb{Z}_{\text {odd }}^{+}\right)$, but the subtlety here is that the perturbation is antiperiodic, from Eq. (34) we have

$$
\cos (\hat{\beta} \phi(x+L))=-\cos (\hat{\beta} \phi(x)),
$$

which is consistent with the fact that its scaling dimension is half-integer and hence in the multi-flavor fermionization it is represented as a product of an odd number of fermions. One 
can modify the definition of the theory at these points by adding a factor of $e^{-i \pi x / L}$ to the tunneling amplitude $\lambda$ to make the perturbation periodic. For the case $\hat{\beta}^{2}=1$ the chiral boson has radius $R_{\mathrm{n}}=1$ and the theory can be fermionized without adding auxiliary degrees of freedom. With the modification to the tunneling amplitude one finds

$$
\begin{aligned}
\mathcal{H}_{\chi S G}^{\hat{\beta}^{2}=1} & =\int_{-L / 2}^{L / 2} d x\left[\frac{1}{4 \pi} v_{\mathrm{n}}:\left(\partial_{x} \phi\right)^{2}:+\frac{\lambda}{\sqrt{2 \pi a}}\left(e^{-i \pi x / L} e^{i \phi(x)}+\text { h.c. }\right)\right] \\
& =\int_{-L / 2}^{L / 2} d x:\left[-i v_{\mathrm{n}} \psi^{\dagger} \partial_{x} \psi+\lambda\left(e^{-i \pi x / L} \psi(x)+e^{i \pi x / L} \psi^{\dagger}(x)\right)\right]: \\
& =\left(\frac{2 \pi v_{\mathrm{n}}}{L}\right) \sum_{r \in \mathbb{Z}+1 / 2} r: c_{r}^{\dagger} c_{r}:+\lambda \sqrt{L}\left(c_{1 / 2}+c_{1 / 2}^{\dagger}\right),
\end{aligned}
$$

where we have used $e^{i \phi(x)} / \sqrt{2 \pi a}=\psi(x)=L^{-1 / 2} \sum_{r} e^{2 \pi i r x / L} c_{r}$. A similar Hamiltonian, with the cosine interaction at a point rather than along the line has appeared in various physical contexts, see for example Ref. [32]. Note that the perturbation changes only a single Fourier mode of the chiral fermion. Therefore in the limit $L \rightarrow \infty$ the perturbation has no effect, which is to be expected since a term in the Hamiltonian consisting of a product of an odd number of fermions cannot produce any off-diagonal long range order.

\section{SEMICLASSICAL CONSIDERATIONS}

In the case of the standard sine-Gordon theory, the classical equation of motion has massive solitonic solutions. A semiclassical analysis about these configurations, valid for small $\hat{\beta}^{2}$, gives information about the spectrum of the quantum theory in the massive phase which exists for $\hat{\beta}^{2}<4$ [33]. In this section we demonstrate that the classical field theory of the $\chi \mathrm{SG}$ model possesses analogous solitary wave (kink) solutions, and we address the question as to whether or not a similar semiclassical expansion is useful.

Consider the $\chi \mathrm{SG}$ model (in the limit $L \rightarrow \infty$ ) as a classical field theory. The real-time Lagrangian density is

$$
\mathcal{L}_{\chi S G}=-\frac{1}{4 \pi} \partial_{x} \phi\left(\partial_{t} \phi+v_{\mathrm{n}} \partial_{x} \phi\right)+\kappa(\cos (\hat{\beta} \phi)-1),
$$

where the classical coupling constant $\kappa$ is related to the dimensionless coupling used in Section III by $\kappa \equiv-\hat{\lambda} /(2 \pi a)^{2}$. We have included a constant term in the potential energy so that the minima at $\phi=2 \pi r / \hat{\beta}, r \in \mathbb{Z}$ have zero energy. The classical equation of motion is

$$
\partial_{x}\left(\partial_{t}+v_{\mathrm{n}} \partial_{x}\right) \phi-2 \pi \kappa \hat{\beta} \sin (\hat{\beta} \phi)=0 .
$$

The conjugate momentum of the field $\phi(x)$ is $\Pi(x)=-\partial_{x} \phi(x) / 4 \pi$, and the Hamiltonian of the theory is

$$
\mathcal{H}_{\chi S G}=\int d x\left[4 \pi v_{\mathrm{n}}(\Pi(x))^{2}-\kappa(\cos (\hat{\beta} \phi)-1)\right]
$$

The equation of motion is reproduced in the Hamiltonian formalism by defining the fundamental Poisson bracket of the chiral Bose field to be [34] 


$$
\left\{\partial_{x} \phi(x), \phi\left(x^{\prime}\right)\right\}_{P B}=2 \pi \delta\left(x-x^{\prime}\right) .
$$

This Poisson bracket is non-canonical, which is to be expected since the system is constrained, i.e., $\Pi(x)$ can be expressed in terms of $\phi(x)$. This is in contrast to the standard SG theory, in which the field and its conjugate momentum are independent dynamical variables. If we define the rescaled field $\bar{\phi} \equiv \hat{\beta} \phi$ to normalize the period of the potential energy term, we see from Eq. (129) that the Poisson bracket of $\partial_{x} \bar{\phi}(x)$ and $\bar{\phi}\left(x^{\prime}\right)$ is proportional to $\hat{\beta}^{2}$. Therefore we would expect a semiclassical analysis to be relevant when $\hat{\beta}^{2}$ is small, just as in the non-chiral SG model. However, recall that the quantum $\chi \mathrm{SG}$ theory at a finite length $L$ is only sensible for $\hat{\beta}^{2} \in \mathbb{Z}$, and hence in the region of $\hat{\beta}^{2}$ where we expect the semiclassical expansion to be most valid, the quantum theory is problematic.

If we consider only static field configurations, the equation of motion is identical to the one obtained from the non-chiral SG theory. Therefore, Eq. (127) has time-independent kink solutions which are identical to those of the standard SG theory. From these stationary configurations we can find time-dependent solutions by applying a boost. The result is:

$$
\phi_{ \pm, v_{s}}(t, x)=\frac{4}{\hat{\beta}} \tan ^{-1}\left\{\exp \left[ \pm \sqrt{\frac{2 \pi \kappa \hat{\beta}^{2}}{v_{\mathrm{n}}-v_{s}}}\left(x-v_{s} t\right)\right]\right\},
$$

where $v_{s} \in\left[-\infty, v_{\mathrm{n}}\right]$ is the velocity of the solitary wave. The topological charge is

$$
N \equiv \frac{\hat{\beta}}{2 \pi} \int d x \partial_{x} \phi(x),
$$

and the solution $\phi_{ \pm, v_{s}}$ has $N= \pm 1$. These solitary waves are not chiral, $v_{s}$ can be positive or negative, but there still is left-right asymmetry. The solitary waves that move to the right (the chirality at $\kappa=0$ ) have a maximum velocity $v_{\mathrm{n}}$ and are contracted relative to the $v_{s}=0$ solutions, while the solitary waves moving to the left have no maximum velocity and are stretched relative to the static solutions. The existence of solitary wave solutions with arbitrarily large velocities is in contrast to the standard SG theory where the solitons have a maximum velocity because of the Lorentz invariance of the theory. Also, in the usual SG theory the solitary wave solutions are solitons. Whether or not this is true in the $\chi \mathrm{SG}$ theory is an open question.

Since $\mathcal{L}_{\chi S G}$ does not depend explicitly on $t$ or $x$ there is a conserved energy, $\mathcal{E}$, and a conserved momentum, $\mathcal{P}$, given by

$$
\begin{aligned}
& \mathcal{E}[\phi]=\int d x\left[\frac{v_{\mathrm{n}}}{4 \pi}\left(\partial_{x} \phi\right)^{2}-\kappa(\cos (\hat{\beta} \phi)-1)\right] \\
& \mathcal{P}[\phi]=\int d x \frac{1}{4 \pi}\left(\partial_{x} \phi\right)^{2}
\end{aligned}
$$

Using Eq. (130) in Eqns. (132) and (133), the energy and momentum of the solitary wave solutions are

$$
\begin{aligned}
& E\left(v_{s}\right) \equiv \mathcal{E}\left[\phi_{ \pm, v_{s}}\right]=\frac{v_{\mathrm{n}} \zeta}{\sqrt{v_{\mathrm{n}}-v_{s}}}+\zeta \sqrt{v_{\mathrm{n}}-v_{s}} \\
& P\left(v_{s}\right) \equiv \mathcal{P}\left[\phi_{ \pm, v_{s}}\right]=\frac{\zeta}{\sqrt{v_{\mathrm{n}}-v_{s}}}
\end{aligned}
$$


where $\zeta \equiv \sqrt{8 \kappa / \pi \hat{\beta}^{2}}$. Note that $P\left(v_{s}\right) \geq 0$ even for solutions that propagate to the left $\left(v_{s}<\right.$ $0)$. The dispersion relation is $E(P)=v_{\mathrm{n}} P+\zeta^{2} / P$, which has a minimum at $P_{0}=\zeta / \sqrt{v_{\mathrm{n}}}$ for which $E\left(P_{0}\right)=2 \sqrt{v_{\mathrm{n}}} \zeta$.

We have found that there are topologically non-trivial solitary wave solutions of the classical equation of motion which exhibit a dispersion relation with a finite energy gap of order $\sqrt{\kappa}$. However, the quantum spectrum of the $\chi \mathrm{SG}$ theory is gapless, at least at the points $\hat{\beta}^{2}=2$ and $\hat{\beta}^{2}=4$. It is unclear how a semiclassical expansion about massive classical solutions could possibly describe the massless quantum theory. A second observation is that while the solitary wave solutions in Eq. (130) conserve the topological charge, the dynamics of the $\chi \mathrm{SG}$ theory does not. From Eqns. (128), (129), and (131) we see that

$$
\left\{N, \mathcal{H}_{\chi S G}\right\}_{P B}=\kappa \hat{\beta}^{2} \int d x \sin \hat{\beta} \phi(x) \text {. }
$$

The functional on the r.h.s. of Eq. (136) is in general non-zero, but it does vanish for $\phi_{ \pm, v_{s}}$ since they are odd functions of $\left(x-v_{s} t\right)$. If at some instant an arbitrarily small perturbation which destroys this symmetry is added to the solitary wave solution, it follows from Eq. (136) that a naive time evolution would result in a non-conserved winding number. However, from Eqns. (131) and (132), we see that if the topological charge of the system departs from an integer value, the energy would be infinite. Thus, if we impose boundary conditions on the field and its spatial derivative such that the energy is finite, specifically,

$$
\lim _{x \rightarrow \pm \infty} \partial_{x} \phi(x)=0, \quad \text { and } \quad \lim _{x \rightarrow \pm \infty} \phi(x) \in\left(\frac{2 \pi}{\hat{\beta}}\right) \mathbb{Z},
$$

then the winding number will be restricted to the integers, and hence it will be conserved if we assume continuity of $\phi(t, x)$. In the quantum $\chi \mathrm{SG}$ theory the topological charge is not conserved, as can be seen from the non-vanishing of the commutator corresponding to the Poisson bracket in Eq. (136). We therefore believe a semiclassical expansion about the solitary wave solutions is unlikely to be useful for understanding the spectrum of the quantum theory.

\section{SUMMARY}

We have considered the edge theory of double-layer quantum Hall systems with a single mode per edge, upon inclusion of interlayer single-electron tunneling at the edge. The theory can be separated into a free chiral boson Hamiltonian for the charged mode and a chiral sine-Gordon Hamiltonian for the neutral mode. We have argued that the RG flow of the $\chi \mathrm{SG}$ theory differs significantly from that of the standard, non-chiral sine-Gordon theory, in particular $\hat{\beta}$ is not renormalized and there is a line of fixed points at $\hat{\beta}^{2}=4$. In addition, the $\chi \mathrm{SG}$ theory likely only makes sense for integer values of $\hat{\beta}^{2}$.

These values in turn break up into an apparently unphysical fermionic sequence, and a bosonic sequence that is realized in quantum Hall systems. In the bosonic sequence there are two points at which tunneling is not irrelevant, $\hat{\beta}^{2}=2$ and $\hat{\beta}^{2}=4$, and each corresponds to an infinite set of double-layer quantum Hall edge theories. For these two cases we have 
obtained exact solutions for the partition function and some correlation functions. At $\hat{\beta}^{2}=2$ we find that tunneling produces spatial oscillations in the correlation functions while at $\hat{\beta}^{2}=4$ tunneling splits the velocities of the two Majorana components of the chiral Dirac fermion corresponding to the neutral mode.

The exact solution of the $\hat{\beta}^{2}=4$ case involves an unfamiliar Bose-Fermi identity, $\psi \partial_{x} \psi \sim$ $e^{2 i \phi}$, which is put on firm ground in the appendices. This is the first in a sequence of identities of the form

$$
e^{i k \phi(x)} \sim \prod_{m=0}^{k-1} \partial_{x}^{m} \psi(x), \quad k \in \mathbb{Z}^{+} .
$$

Extensions of this work to include the effects of disorder, asymmetric velocities $v \neq v^{\prime}$, and the case of more than two layers are currently being pursued [9] and we expect to discuss these, along with a proposal to detect the trifurcation of the electron at the 331 edge, in a future publication.

\section{ACKNOWLEDGMENTS}

We would like to thank E. Fradkin, I. Gruzberg, I. Klebanov, R. Konik, D.-H. Lee, M. Stone, H. Verlinde, and X.-G. Wen for useful discussions. We thank H. Saleur for bringing Ref. [5] to our attention. We would like to acknowledge support by a NSF Graduate Research Fellowship (JDN), DOE Grant DE-FG02-90ER40542 (LPP) as well as NSF grant No. DMR96-32690, US-Israel BSF grant No. 9600294, and fellowships from the A. P. Sloan Foundation and the David and Lucille Packard Foundation (SLS).

Note added: After completion of this work, F. D. M. Haldane pointed out to us that identities similar to Eq. (68) are implicit in work involving Umklapp scattering for spinless electrons in one dimension [35,36]. As we have been unable to find an explicit proof in the literature we have chosen to retain our proofs in the interests of completeness.

\section{APPENDIX A: KLEIN FACTORS}

In this appendix we demonstrate that it is possible to modify our definitions of fermionic vertex operators in a way that gives the proper anticommutation relations between different flavors without altering the form of the Hamiltonian. The first case to consider is the physical electron operators defined in Eq. (25), which commute for electrons in different layers. We modify this definition to

$$
\Psi_{i}(x)=\frac{1}{L^{m / 2}} e^{i \pi S_{i j} \mathcal{N}_{j}} e^{i u_{i}(x)},
$$

where $S$ is a matrix used to enforce the correct anticommutation relations. We now have, using Eqns. (7) and (10),

$$
\Psi_{i}(x) \Psi_{j}\left(x^{\prime}\right)=\Psi_{j}\left(x^{\prime}\right) \Psi_{i}(x) e^{i \pi\left[\operatorname{sgn}\left(x^{\prime}-x\right) K_{i j}-S_{i k} K_{k j}+S_{j k} K_{k i}\right]} \quad \text { for } \quad x \neq x^{\prime} .
$$


If $K_{12}=n$ is odd we can set $S=0$ and have the correct anticommutation relations between the electron operators in different layers. If $n$ is even then we require

$$
n\left(S_{11}-S_{22}\right)+m^{\prime} S_{12}-m S_{21} \in \mathbb{Z}_{\text {odd }}
$$

From the dimension of the operator in Eq. (A1) it is clear that for $m \neq 1$ it is not possible for $\Psi_{i}(x)$ and $\Psi_{i}^{\dagger}\left(x^{\prime}\right)$ to have a canonical anticommutator; a characteristic consequence of a low-energy projection. It can be shown that $\left\{: \Psi_{i}(x):,: \Psi_{j}^{\dagger}\left(x^{\prime}\right):\right\} \propto \delta_{i j} \delta^{(m-1)}\left(x-x^{\prime}\right)$ in the limit $a \rightarrow 0, L \rightarrow \infty$.

Using Eq. (A1) in the definition of the tunneling Hamiltonian (29) gives:

$$
\mathcal{H}_{1}=\frac{\lambda_{0}}{L^{m}} \int_{-L / 2}^{L / 2} d x\left(: e^{i \pi\left(S_{2 j}\left(K_{j 2}-K_{j 1}\right)-\left(S_{1 j}-S_{2 j}\right) \mathcal{N}_{j}\right)} e^{i u_{1}(x)} e^{-i u_{2}(x)}:+ \text { h.c. }\right) .
$$

The topological-charge dependent coefficient is just \pm 1 provided:

$$
S_{12}-S_{22}, \quad S_{11}-S_{21} \in \mathbb{Z}_{\text {even }}
$$

For any even $n$ and odd $m, m^{\prime}$ there are infinitely many choices for $S$ that simultaneously satisfy Eq. (A3) and Eq. (A5). We make the particularly simple choice

$$
S=\left(\begin{array}{ll}
1 & 0 \\
1 & 0
\end{array}\right) \quad \text { for } \quad n \in \mathbb{Z}_{\text {even }},
$$

and absorb the overall sign into the definition of the tunneling amplitude $\lambda \rightarrow(-1)^{n+1} \lambda$, making Eq. (A4) identical to Eq. (29).

The second place where Klein factors are needed is in the fermionizations of the $\chi \mathrm{SG}$ Hamiltonian in Section $\nabla$. For the case $\hat{\beta}^{2}=2$, Eq. (88) should be modified to read

$$
\psi_{i}(x)=\frac{1}{\sqrt{2 \pi a}} e^{i \pi S_{i j} N_{j}^{\theta}} e^{i \theta_{i}(x)} .
$$

The matrix $S$ is again given by Eq. (A6) and ensures the proper anticommutation relations between the different fermion species without modifying Eq. (89), just as in Eq. (A4). For the multi-flavor fermionization at $\hat{\beta}^{2}=4$, Eq. (105) should be replaced by

$$
\psi_{i}(x)=\frac{1}{\sqrt{2 \pi a}} e^{i \pi P_{i j} N_{j}^{\theta}} e^{i \theta_{i}(x)}
$$

where the matrix $P$ is chosen so that $\psi_{i}$ and $\psi_{j}$ anticommute for $i \neq j$. One can readily show:

$$
\psi_{i}(x) \psi_{j}\left(x^{\prime}\right)=\psi_{j}\left(x^{\prime}\right) \psi_{i}(x) e^{i \pi\left(P_{j i}-P_{i j}+\operatorname{sgn}\left(x-x^{\prime}\right) \delta_{i j}\right)} \quad \text { for } \quad x \neq x^{\prime}
$$

and therefore we demand

$$
P_{i j}-P_{j i} \in \mathbb{Z}_{\text {odd }} \quad \text { for } \quad i \neq j .
$$

Using Eq. (A8) in Eq. (103) we find the fermionized Hamiltonian including Klein factors is 


$$
\begin{gathered}
\hat{\mathcal{H}}_{\chi S G}^{\hat{\beta}^{2}=4}=\int_{-L / 2}^{L / 2} d x:\left[-i v_{\mathrm{n}}^{\prime} \psi_{i}^{\dagger} \partial_{x} \psi_{i}+\lambda\left(e^{i \pi p} e^{i \pi O_{i} P_{i j} N_{j}} \psi_{1} \psi_{2}^{\dagger} \psi_{3} \psi_{4}^{\dagger}+\text { h.c. }\right)\right. \\
\left.+\frac{\lambda}{2}\left(\psi_{1}^{\dagger} \psi_{1}-\psi_{2}^{\dagger} \psi_{2}\right)\left(\psi_{4}^{\dagger} \psi_{4}-\psi_{3}^{\dagger} \psi_{3}\right)\right]:
\end{gathered}
$$

where $O_{i}=(-1)^{i}$ and $p=P_{21}-P_{22}+P_{32}-P_{31}+P_{41}-P_{42}+P_{43}-P_{44}$. From Eqns. (A10) and (A11) we see that one choice of $P$ that gives the correct anticommutation relations and which trivializes the explicit topological charge dependence of the Hamiltonian is

$$
P=\left(\begin{array}{llll}
0 & 1 & 1 & 1 \\
0 & 1 & 1 & 1 \\
0 & 0 & 0 & 1 \\
0 & 0 & 0 & 1
\end{array}\right)
$$

With this choice of $P$, we find Eq. (A11) is identical to Eq. (106).

\section{APPENDIX B: EQUIVALENCE OF CORRELATION FUNCTIONS}

In this appendix we prove the validity of the identity

$$
\psi(x) \partial_{x} \psi(x)=\frac{i}{2 \pi a^{2}} e^{2 i \phi(x)},
$$

by comparing the $2 N$-point correlation functions of these operators and their Hermitian conjugates. We work at zero temperature for simplicity. Consider first the correlation function in the free chiral boson theory:

$$
\begin{aligned}
\mathcal{B}_{N}\left(z_{1}, \ldots, z_{N} ; z_{1}^{\prime}, \ldots, z_{N}^{\prime}\right) & \equiv\left\langle\prod_{i=1}^{N}\left(\frac{i}{2 \pi a^{2}}\right) e^{2 i \phi\left(\tau_{i}, x_{i}\right)} \prod_{i=1}^{N}\left(\frac{-i}{2 \pi a^{2}}\right) e^{-2 i \phi\left(\tau_{i}^{\prime}, x_{i}^{\prime}\right)}\right\rangle \\
& =\frac{1}{(2 \pi)^{2 N}} \frac{\prod_{1 \leq i<j \leq N}\left(z_{i}-z_{j}\right)^{4}\left(z_{i}^{\prime}-z_{j}^{\prime}\right)^{4}}{\prod_{1 \leq i, j \leq N}\left(z_{i}-z_{j}^{\prime}\right)^{4}} .
\end{aligned}
$$

From Eq. (B1), the corresponding correlation function in the free chiral fermion theory is:

$$
\mathcal{F}_{N}\left(z_{1}, \ldots, z_{N} ; z_{1}^{\prime}, \ldots, z_{N}^{\prime}\right) \equiv\left\langle\prod_{i=1}^{N} \psi\left(\tau_{i}, x_{i}\right) \partial_{x_{i}} \psi\left(\tau_{i}, x_{i}\right) \prod_{i=1}^{N}\left(-\psi^{\dagger}\left(\tau_{i}^{\prime}, x_{i}^{\prime}\right) \partial_{x_{i}^{\prime}} \psi^{\dagger}\left(\tau_{i}^{\prime}, x_{i}^{\prime}\right)\right)\right\rangle .
$$

We adopt the complex notation $\psi(\tau, x) \equiv \psi(z), \partial_{x} \psi(\tau, x) \equiv-i \partial_{z} \psi(z)$. To evaluate this $2 N$-point function we write it as the limit of a $4 N$-point function and pull the derivatives outside the correlator:

$$
\begin{aligned}
\mathcal{F}_{N}\left(\left\{z_{i}\right\},\left\{z_{i}^{\prime}\right\}\right)= & \lim _{w_{1}, w_{2} \rightarrow z_{1}} \lim _{w_{1}^{\prime}, w_{2}^{\prime} \rightarrow z_{1}^{\prime}} \cdots \lim _{w_{2 N-1}, w_{2 N} \rightarrow z_{N}} \lim _{w_{2 N-1}^{\prime}, w_{2 N}^{\prime} \rightarrow z_{N}^{\prime}} \\
& {\left[\prod_{j=1}^{N}\left(\partial_{w_{2 j}} \partial_{w_{2 j}^{\prime}}\right)\left\langle\prod_{i=1}^{2 N} \psi\left(w_{i}\right) \prod_{i=1}^{2 N} \psi^{\dagger}\left(w_{i}^{\prime}\right)\right\rangle\right] . }
\end{aligned}
$$

From Eqns. (80) and (81) we find that the $4 N$-point correlation function can be written: 


$$
\left\langle\prod_{i=1}^{2 N} \psi\left(w_{i}\right) \prod_{i=1}^{2 N} \psi^{\dagger}\left(w_{i}^{\prime}\right)\right\rangle=\frac{1}{(2 \pi)^{2 N}} \frac{\prod_{1 \leq i<j \leq 2 N}\left(w_{i}-w_{j}\right)\left(w_{i}^{\prime}-w_{j}^{\prime}\right)}{\prod_{1 \leq i, j \leq 2 N}\left(w_{i}-w_{j}^{\prime}\right)}
$$

We first organize the products appearing in Eq. (B5) by separating coordinates, $w_{i}$, with even and odd indices. The denominator can be expressed as

$$
\prod_{i=1}^{2 N} \prod_{j=1}^{2 N}\left(w_{i}-w_{j}^{\prime}\right)=\prod_{i=1}^{N} \prod_{j=1}^{N}\left[\left(w_{2 i-1}-w_{2 j-1}^{\prime}\right)\left(w_{2 i}-w_{2 j-1}^{\prime}\right)\left(w_{2 i-1}-w_{2 j}^{\prime}\right)\left(w_{2 i}-w_{2 j}^{\prime}\right)\right],
$$

and the numerator can be rewritten using

$$
\begin{aligned}
\prod_{i=1}^{2 N-1} \prod_{j=i+1}^{2 N}\left(w_{i}-w_{j}\right)= & \prod_{i=1}^{N-1} \prod_{j=i+1}^{N}\left[\left(w_{2 i-1}-w_{2 j-1}\right)\left(w_{2 i}-w_{2 j-1}\right)\right. \\
& \left.\times\left(w_{2 i-1}-w_{2 j}\right)\left(w_{2 i}-w_{2 j}\right)\right] \prod_{k=1}^{N}\left(w_{2 k-1}-w_{2 k}\right)
\end{aligned}
$$

and an identical expression with $w_{i}$ replaced by $w_{i}^{\prime}$.

When the $N^{2}$ derivatives in Eq. (B4) act on the expression on the r.h.s. of Eq. (B5), a large number of terms are generated. However, from Eq. (B7) we see that the numerator in Eq. (B5) contains a factor $\left(w_{2 k-1}-w_{2 k}\right)$ for each $k=1, \ldots N$. In the limit $w_{2 k-1}, w_{2 k} \rightarrow z_{k}$, this factor vanishes, and hence the only term that will be non-zero in the multiple limit given in Eq. (B4) is the term in which the operator $\partial_{w_{2 k}}$ acts on $\left(w_{2 k-1}-w_{2 k}\right)$ for all $k=1, \ldots N$. An analogous argument holds for the primed coordinates and therefore we find using Eqns. (B2), and (B4)-(B7):

$$
\begin{aligned}
& \mathcal{F}_{N}\left(\left\{z_{i}\right\},\left\{z_{i}^{\prime}\right\}\right)=\lim _{w_{1}, w_{2} \rightarrow z_{1}} \lim _{w_{1}^{\prime}, w_{2}^{\prime} \rightarrow z_{1}^{\prime}} \cdots \lim _{w_{2 N-1}, w_{2 N} \rightarrow z_{N}} \lim _{w_{2 N-1}^{\prime}, w_{2 N}^{\prime} \rightarrow z_{N}^{\prime}} \\
& \times \frac{1}{(2 \pi)^{2 N}} \frac{\prod_{1 \leq i<j \leq N}\left[\left(w_{2 i-1}-w_{2 j-1}\right)\left(w_{2 i}-w_{2 j-1}\right)\left(w_{2 i-1}-w_{2 j}\right)\left(w_{2 i}-w_{2 j}\right)\right] \times\left[w_{i} \rightarrow w_{i}^{\prime}\right]}{\prod_{1 \leq i, j \leq N}\left(w_{2 i-1}-w_{2 j-1}^{\prime}\right)\left(w_{2 i}-w_{2 j-1}^{\prime}\right)\left(w_{2 i-1}-w_{2 j}^{\prime}\right)\left(w_{2 i}-w_{2 j}^{\prime}\right)} \\
& =\frac{1}{(2 \pi)^{2 N}} \frac{\prod_{1 \leq i<j \leq N}\left(z_{i}-z_{j}\right)^{4}\left(z_{i}^{\prime}-z_{j}^{\prime}\right)^{4}}{\prod_{1 \leq i, j \leq N}\left(z_{i}-z_{j}^{\prime}\right)^{4}}=\mathcal{B}_{N}\left(\left\{z_{i}\right\},\left\{z_{i}^{\prime}\right\}\right),
\end{aligned}
$$

completing the proof.

\section{APPENDIX C: EQUIVALENCE OF COMMUTATION RELATIONS}

Here we calculate some additional commutation relations in support of the identification (68), which can be written in terms of a normal-ordered exponential [using Eq. ([19)] as

$$
\frac{2 \pi i}{L^{2}}: e^{2 i \phi}:=\psi \partial_{x} \psi
$$

Consider the operators 


$$
\begin{aligned}
\mathbf{A}^{(p-1)}(x) & \equiv \frac{1}{p k_{1}^{p}}:\left(\partial_{x} \phi\right)^{p}:=\sum_{n} \mathbf{A}_{n}^{(p-1)} e^{i k_{n} x} \\
\mathbf{C}^{( \pm)}(x) & \equiv \frac{1}{2}:\left(e^{2 i \phi} \pm e^{-2 i \phi}\right):=\sum_{n} \mathbf{C}_{n}^{( \pm)} e^{i k_{n} x}
\end{aligned}
$$

where $k_{1} \equiv 2 \pi / L$ and the rightmost sides of the expressions define the normalization of the associated harmonics. One can check that

$$
\begin{aligned}
{\left[\mathbf{C}_{n}^{( \pm)}, \mathbf{A}_{m}^{(0)}\right] } & =-2 \mathbf{C}_{n+m}^{(\mp)} \\
{\left[\mathbf{C}_{n}^{( \pm)}, \mathbf{A}_{m}^{(1)}\right] } & =(n-m) \mathbf{C}_{n+m}^{( \pm)} \\
{\left[\mathbf{C}_{n}^{( \pm)}, \mathbf{C}_{m}^{( \pm)}\right] } & =(n-m) \mathbf{A}_{n+m}^{(1)}+\frac{1}{12} n\left(n^{2}-1\right) \delta_{n+m} \\
{\left[\mathbf{C}_{n}^{(+)}, \mathbf{C}_{m}^{(-)}\right] } & =2 \mathbf{A}_{n+m}^{(2)}+\frac{n^{2}-n m+m^{2}-1}{6} \mathbf{A}_{n+m}^{(0)}
\end{aligned}
$$

For example, to derive Eq. (C6), we write each operator as a Fourier transformation,

$$
\mathbf{C}_{n}^{( \pm)}=L^{-1} \int_{-L / 2}^{L / 2} d x e^{-i k_{n} x} \mathbf{C}^{( \pm)}(x)
$$

and evaluate the products of normal ordered vertex operators with the help of decomposition (31) explicitly at a given cutoff $a>0$,

$$
: e^{i \alpha_{1} \phi(x)}:: e^{i \alpha_{2} \phi(y)}:=: e^{i \alpha_{1} \phi(x)+i \alpha_{2} \phi(y)}: \frac{e^{-\pi i \alpha_{1} \alpha_{2}(y-x) / L}}{\left[1-e^{2 \pi i(y-x+i a) / L}\right]^{-\alpha_{1} \alpha_{2}}}
$$

In the limit $a \rightarrow 0$, the commutator of these two vertex operators vanishes at $x \neq y$ for even integer values of the product $\alpha_{1} \alpha_{2}$, but it has a pole of order $\left|\alpha_{1} \alpha_{2}\right|$ at $x=y$ for negative values of the product, $\alpha_{1} \alpha_{2}<0$. A direct calculation with the help of the substitution $z=\exp (2 \pi i x / L)$ shows that the only contribution to the commutator (C6) is given by the corresponding residue,

$$
\begin{aligned}
{\left[\mathbf{C}_{n}^{(+)}, \mathbf{C}_{m}^{(+)}\right] } & =\left.\frac{1}{4} \sum_{\alpha= \pm 2} \oint_{|z|=1} \frac{d z z^{\alpha^{2} / 2-n-1}}{(2 \pi i)\left(\alpha^{2}-1\right) !} \frac{\partial^{\left(\alpha^{2}-1\right)}}{\partial w^{\left(\alpha^{2}-1\right)}}\left[w^{\alpha^{2} / 2-m-1}: e^{i \alpha\left(\varphi_{z}-\varphi_{w}\right)}:\right]\right|_{w=z} \\
& =\left.\frac{1}{24} \sum_{\alpha= \pm 2} \oint \frac{d z z^{1-n}}{2 \pi i} \frac{\partial^{3}}{\partial w^{3}}\left[w^{1-m}: e^{i \alpha\left(\varphi_{z}-\varphi_{w}\right)}:\right]\right|_{w=z} \\
= & \oint_{|z|=1} \frac{d z z^{-n-m-1}}{2 \pi i}\left[\frac{m\left(1-m^{2}\right)}{12}+(m-1) z^{2}:\left(\partial_{z} \varphi_{z}\right)^{2}:-\frac{z^{3}}{2} \partial_{z}:\left(\partial_{z} \varphi_{z}\right)^{2}:\right]
\end{aligned}
$$

and a simple integration by parts gives the result (C6). In Eq. (C10) we used [cf. Eq. (177)]

$$
\varphi_{z}=-N R i \ln z-\chi / R+\sum_{r=1}^{\infty} \frac{1}{\sqrt{r}} e^{-k_{r} a / 2}\left(z^{-r} b_{r}^{\dagger}+z^{r} b_{r}\right) .
$$


Readers familiar with conformal field theory techniques will notice that Eq. (C9) could be obtained immediately within the formalism of canonical quantization on a cylinder [25]. We also notice that Eq. (C5) follows from a more general relationship for the vertex operator $\mathcal{V}^{\alpha}(x) \equiv: e^{i \alpha \phi}:$

$$
\left[\mathcal{V}_{n}^{\alpha}, \mathbf{A}_{m}^{(1)}\right]=\left(n+m-m \frac{\alpha^{2}}{2}\right) \mathcal{V}_{n+m}^{\alpha},
$$

which can be derived in a variety of standard ways.

On the other hand, using Eq. (C1) and the canonical bosonization prescription, we can define the fermionic counterparts of the same operators,

$$
\begin{aligned}
\tilde{\mathbf{A}}^{(p-1)}(x) & \equiv \frac{L^{p}}{p}:\left(: \psi^{\dagger} \psi:\right)^{p}: \\
\tilde{\mathbf{C}}^{( \pm)}(x) & \equiv \frac{L^{2}}{4 \pi i}\left(\psi \partial_{x} \psi \mp \partial_{x} \psi^{\dagger} \psi^{\dagger}\right)=\frac{1}{2} \sum_{r} e^{i k_{r} x} \sum_{s} s\left(c_{r-s} c_{s} \pm c_{s}^{\dagger} c_{s-r}^{\dagger}\right),
\end{aligned}
$$

where the canonical Fermi operators $c_{r},\left\{c_{r}, c_{s}^{\dagger}\right\}=\delta_{r s}$ are defined by the expansion of the antiperiodic field

$$
\psi(x)=L^{-1 / 2} \sum_{r \in \mathbb{Z}+\frac{1}{2}} c_{r} e^{2 \pi i r x / L} .
$$

Comparing their commutation relations, it is a straightforward (but lengthy) exercise to express the operators

$$
\tilde{\mathbf{A}}_{n}^{(0)}=\tilde{\mathbf{R}}_{n}^{(0,0)}, \quad \tilde{\mathbf{A}}_{n}^{(1)}=\frac{1}{2}\left(\tilde{\mathbf{R}}_{n}^{(1,0)}+\tilde{\mathbf{R}}_{n}^{(0,1)}\right), \quad \tilde{\mathbf{A}}_{n}^{(2)} \equiv \tilde{\mathbf{R}}_{n}^{(1,1)}+\frac{2 n^{2}+1}{6} \tilde{\mathbf{R}}_{n}^{(0,0)}, \ldots
$$

in terms of operators

$$
\tilde{\mathbf{R}}_{n}^{(a, b)}=\lim _{K \rightarrow \infty} \sum_{r=-K}^{K+n}(r-n)^{a} r^{b}: c_{r-n}^{\dagger} c_{r}:
$$

bilinear in fermions. The commutation relations then read

$$
\begin{aligned}
{\left[\tilde{\mathbf{C}}_{n}^{( \pm)}, \tilde{\mathbf{A}}_{m}^{(0)}\right] } & =-2 \tilde{\mathbf{C}}_{n+m}^{(\mp)}, \\
{\left[\tilde{\mathbf{C}}_{n}^{( \pm)}, \tilde{\mathbf{A}}_{m}^{(1)}\right] } & =(n-m) \tilde{\mathbf{C}}_{n+m}^{( \pm)}, \\
{\left[\tilde{\mathbf{C}}_{n}^{( \pm)}, \tilde{\mathbf{C}}_{m}^{( \pm)}\right] } & =(n-m) \tilde{\mathbf{A}}_{n+m}^{(1)}+\frac{1}{12} n\left(n^{2}-1\right) \delta_{n+m}, \\
{\left[\tilde{\mathbf{C}}_{n}^{(+)}, \tilde{\mathbf{C}}_{m}^{(-)}\right] } & =2 \tilde{\mathbf{R}}_{n+m}^{(1,1)}+\frac{(n+m)^{2}-n m}{2} \tilde{\mathbf{R}}_{n+m}^{(0,0)} \\
& =2 \tilde{\mathbf{A}}_{n+m}^{(2)}+\frac{n^{2}-n m+m^{2}-1}{6} \tilde{\mathbf{A}}_{n+m}^{(0)} ;
\end{aligned}
$$

they are in precise correspondence with Eqns. (C4)-(C7). 


\section{APPENDIX D: ALTERNATIVE SOLUTION OF THE $\chi$ SG THEORY AT $\hat{\beta}^{2}=4$.}

With the help of the commutation relations derived in Appendix Q , we can diagonalize the $\chi$ SG Hamiltonian at $\hat{\beta}^{2}=4$, Eq. (65), directly within the operator formalism. Indeed, in terms of normal-ordered quantities the Hamiltonian reads

$$
\begin{aligned}
\mathcal{H}_{\chi S G}^{\hat{\beta}^{2}=4} & =\int_{-L / 2}^{L / 2} d x\left[\frac{v_{\mathrm{n}}}{4 \pi}:\left(\partial_{x} \phi\right)^{2}:+\frac{2 \lambda}{L^{2}}: \cos (2 \phi):\right] \\
& =\frac{2 \pi}{L^{2}} \int_{-L / 2}^{L / 2} d x\left[v_{\mathrm{n}} A^{(1)}(x)+\frac{\lambda}{\pi} C^{(+)}(x)\right] \\
& =\frac{2 \pi}{L}\left[v_{\mathrm{n}} A_{0}^{(1)}+\frac{\lambda}{\pi} C_{0}^{(+)}\right]
\end{aligned}
$$

where we used the normal ordering formula (19) and the definitions (C2), (C3); the last line is written as a sum of two commuting operators. The commutation relations (C4)-(C7) imply that the operators

$$
L_{n}^{ \pm} \equiv \frac{1}{2}\left(A_{n} \pm C_{n}^{(+)}\right)
$$

generate two independent $c=1 / 2$ Virasoro algebras,

$$
\left[L_{n}^{a}, L_{m}^{b}\right]=\delta^{a b}(n-m) L_{n+m}^{b}+\frac{1}{24} n\left(n^{2}-1\right) \delta_{n+m, 0} \delta^{a b}
$$

and the Hamiltonian (D2) becomes

$$
\mathcal{H}_{\chi S G}^{\hat{\beta}^{2}=4}=(2 \pi / L)\left(v_{+} L_{0}^{+}+v_{-} L_{0}^{-}\right), \quad v_{ \pm}=v_{\mathrm{n}} \pm \lambda / \pi
$$

Now the spectrum can be obtained directly in terms of irreducible representations of the two $c=1 / 2$ Virasoro algebras. To this end it is convenient to write each set of operators $L_{n}^{a}, a= \pm$, in terms of an independent Majorana fermion. In particular,

$$
\frac{2 \pi}{L} L_{0}^{a}=\frac{1}{2 i} \int_{-L / 2}^{L / 2} d x: \chi_{a} \partial_{x} \chi_{a}:
$$

which takes us back to the Hamiltonian (70) obtained by a more conventional fermionization procedure. 


\section{REFERENCES}

[1] S. R. Renn, Phys. Rev. Lett. 68 (1992) 658.

[2] C. L. Kane, M. P. A. Fisher, and J. Polchinski, Phys. Rev. Lett. 72 (1994) 4129.

[3] F. D. M. Haldane., Phys. Rev. Lett. 74 (1995) 2090.

[4] J. E. Moore and X.-G. Wen, Phys. Rev. B 57 (1998) 10138.

[5] R. Dijkgraaf, Nucl. Phys. B 493 (1997) 588.

[6] J. Cardy, Nucl. Phys. B 389 (1993) 577.

[7] R. Jackiw and R. Rajaraman, Phys. Rev. Lett. 54 (1985) 1219.

[8] A. F. Ho and P. Coleman, cond-mat/9812441; cond-mat/9903197.

[9] J. D. Naud, L. P. Pryadko, and S. L. Sondhi, unpublished.

[10] B. I. Halperin, Helv. Phys. Acta 56 (1983) 75.

[11] S. M. Girvin and A. H. MacDonald, in Novel Quantum Liquids in Low-Dimensional Semiconductor Structures, ed. S. DasSarma and A. Pinczuk (Wiley, New York, 1995).

[12] X.-G. Wen, Phys. Rev. B 43 (1991) 11025; Phys. Rev. Lett. 64 (1990) 2206; Int. J. Mod. Phys. B 6 (1992) 1711; Adv. in Phys. 44 (1995) 405.

[13] X.-G. Wen and A. Zee, Phys. Rev. B 46 (1992) 2290.

[14] M. Milovanović and N. Read, Phys. Rev. B 53 (1996) 13559.

[15] N. P. Sandler, C. de C. Chamon, E. Fradkin, Phys. Rev. B 57 (1998) 12324.

[16] C. L. Kane and M. P. A. Fisher, Phys. Rev. B 51 (1995) 13449.

[17] M. Stone, Ann. of Phys. 207 (1991) 38.

[18] J. von Delft and H. Schoeller, Annalen Phys. 7 (1998) 225.

[19] R. Floreanini and R. Jackiw, Phys. Rev. Lett. 59 (1987) 1873.

[20] S. Coleman, Phys. Rev. D 11 (1975) 2088.

[21] J. Kogut, Rev. of Mod. Phys. 51 (1979) 659.

[22] P. B. Wiegmann, J. Phys. C 11 (1978) 1583.

[23] T. Ohta, Prog. of Theor. Phys. 60 (1978) 968.

[24] I. Ichinose and H. Mukaida, Int. J. Mod. Phys. A 9 (1994) 1043.

[25] P. Di Francesco, P. Mathieu, and D. Sénéchal, Conformal Field Theory (Springer, New York, 1997).

[26] P. Goddard, A. Kent, and D. Olive, Comm. Math. Phys. 103 (1986) 105.

[27] P. Ginsparg, in Fields, Strings, and Critical Phenomena, Les Houches Session XLIX, ed. E. Brézin and J. Zinn-Justin (North-Holland, Amsterdam, 1989).

[28] K. Nomura and D. Yoshioka, cond-mat/9904192.

[29] A. M. Finkel'stein, and A. I. Larkin, Phys. Rev. B 47 (1993) 10461.

[30] J. K. Jain, Phys. Rev. B 40 (1989) 8079.

[31] Bateman Manuscript Project, Higher Transcendental Functions, Vol. II (Krieger, Malabar, 1981).

[32] K. A. Matveev, Phys. Rev. B 51 (1994) 1743.

[33] R. Rajaraman, Solitons and Instantons (North-Holland, Amsterdam, 1982).

[34] F. D. M. Haldane, unpublished.

[35] F. D. M. Haldane, Phys. Rev. Lett. 45 (1980) 1358.

[36] J. L. Black and V. J. Emery, Phys. Rev. B 23 (1981) 429. 